\title{
ONE DIMENSIONAL STEFAN PROBLEMS WITH NONMONOTONE FREE BOUNDARY
}

\author{
BY \\ AVNER FRIEDMAN( $\left.{ }^{1}\right)$
}

Introduction. In a recent paper [3] we have considered the Stefan problem in the case of an arbitrary space-dimension $n$. We have proved existence, uniqueness, stability and asymptotic theorems for a weak solution. As a by-product we have obtained the following results for a two-phase problem in the case $n=1$ :

(1) The weak solution $u(x, t)$ is a continuous function.

(2) If the boundary data converge at $t=\infty$ in a suitable way, then $u(x, t) \rightarrow w(x)$ uniformly with respect to $x$, where $w(x)$ is the solution of the appropriate stationary problem.

The weak solution of [3] is a classical solution of the appropriate parabolic equation in every open set where $u \geqq 0$ and $a(u) \geqq 0$ (or where $u \leqq 0$ and $a(u) \leqq-\alpha$ ). However the set $W$ where $u=0$ and $-\alpha<a(u)<0$ was not investigated in [3] except for the fact that (for any $n \geqq 1$ ) $W$ stays away from the fixed boundaries. It is not known, for instance, whether or not $W$ has a positive measure or interior points. For the one-phase problem, however, it was proved in [3] that $W$ is contained in the boundary of measurable point and, consequently, has no interior points. Physically, $W$ represents the "weak" free boundary between the solid and the liquid of the problem.

The first purpose of the present paper is to prove that, in the case $n=1, W$ is actually a continuous curve $x=s(t)$ (which is then the free boundary in the usual sense) and that $u(x, t) \neq 0$ at all the points where $x \neq s(t)$. This result is stated in Theorem 3 below, and is proved in $\$ \S 2,3$. The second purpose of the paper is to extend the results from two-phase problems to $k$-phase problems for any $k$. This is done in $\$ \S 5-7$. The lines $t=\sigma_{i}$ where some of the phases degenerate cause some difficulty. There may occur perhaps some intervals on $t=\sigma_{i}$ where $u\left(x, \sigma_{i}\right) \equiv 0$ (see §6) and regions lying above such intervals where $u \equiv 0$ (see \$7). But in any case we prove that $W$ consists of a finite number of curves $x=x(t)$ which are continuous in the intervals $\left(\sigma_{i}, \sigma_{i+1}\right)$.

As far as we know, global existence theorems of classical solutions for the twophase Stefan problem were considered only by Rubinstein [4]. However, there is a fundamental gap in his proof of existence. In $\$ 8$ we explain the nature of this gap. Thus it is not known, at this point, whether classical solutions do in fact exist globally.

Received by the editors January 27, 1967.

(') This work was supported by the Guggenheim Fellowship and by the National Science Foundation NSF Grant GP-5558. 
In $\$ 8$ we also give some generalizations of the results of the previous sections.

1. The main results for the 2-phase problem. Consider the system of equations

$$
\begin{array}{ll}
\frac{\partial u_{i}}{\partial t}=\beta_{i} \frac{\partial^{2} u_{i}}{\partial x^{2}} & \text { for } b_{1}<x<s(t), \quad 0<t<T \text { if } i=1 \\
\text { and } s(t)<x<b_{2}, & 0<t<T \text { if } i=2
\end{array}
$$

where $\beta_{i}>0$,

$$
u_{i}\left(b_{i}, t\right)=g_{i}(t) \quad \text { for } 0 \leqq t<T \quad(i=1,2)
$$

where $g_{1}>0, g_{2}<0$,

$$
\begin{aligned}
& u_{i}(x, 0)=h_{t}(x) \quad \begin{array}{l}
\text { for } b_{1}<x<b \text { if } i=1, \\
\text { and } b<x<b_{2} \text { if } i=2,
\end{array}
\end{aligned}
$$

where $h_{1}>0, h_{2}<0, s(0)=b$,

$$
\begin{gathered}
u_{i}(s(t), t)=0 \quad \text { for } 0 \leqq t<T \quad(i=1,2), \\
\beta_{1} \partial u_{1} / \partial x-\beta_{2} \partial u_{2} / \partial x=-\alpha d s / d t \quad \text { for } x=s(t), \quad 0<t<T
\end{gathered}
$$

where $\alpha>0$. Given $g_{i}, h_{i}, b$, the classical two-phase Stefan problem consists of finding $\left(u_{1}, u_{2}, s\right)$ such that (1.1)-(1.5) hold. It is required that all the derivatives of $u_{1}, u_{2}, s$ which occur in (1.1)-(1.5) exist in the usual sense and are continuous in the appropriate regions (compare [1]). We then call $\left(u_{1}, u_{2}, s\right)$ a classical solution.

As proved in [1], a classical solution of (1.1)-(1.5) is known to exist locally (i.e. for $0 \leqq t<\varepsilon, \varepsilon$ sufficiently small) provided $g_{i}$ and $h_{i}$ satisfy some smoothness assumptions.

Set $\alpha_{i}=1 / \beta_{i}$ and introduce

$$
\begin{aligned}
a(u) & =\alpha_{2} u-\alpha & & \text { if } u \leqq 0, \\
& =\alpha_{1} u & & \text { if } u>0, \\
g & =g_{1} / \alpha_{1} & & \text { on } x=b_{1}, \\
& =g_{2} / \alpha_{2} & & \text { on } x=b_{2}, \\
h & =h_{1} / \alpha_{1} & & \text { for } b_{1}<x<b, \\
& =h_{2} / \alpha_{2} & & \text { for } b<x<b_{2}, \\
& =0 & & \text { for } x=b .
\end{aligned}
$$

Finally we set $G=\left\{x ; b_{1}<x<b_{2}\right\}, \Omega_{T}=G \times\{0<t<T\}$.

Definition. A bounded measurable function $u(x, t)$ on $\Omega_{T}$ is called a weak solution of (1.1)-(1.5) if the equality

$$
\iint_{\Omega_{T}}\left[u \phi_{x x}+a(u) \phi_{t}\right] d x d t=\int_{0}^{T}\left[g \phi_{x}\right]_{x=b_{1}}^{x=b_{2}} d t-\int_{G} a(h) \phi(x, 0) d x
$$

holds for any $\phi$ such that $\phi_{x}, \phi_{x x}, \phi_{t}$ are continuous in $\Omega_{T}$ and $\phi=0$ on $x=b_{1}$, $x=b_{2}$ and on $t=T$. Here, the function $a(u(x, t))$ on the set where $u(x, t)=0$ is 
defined as an arbitrary function $\beta(x, t)$ subject to the following conditions: (i) $-\alpha \leqq \beta(x, t) \leqq 0$, (ii) $a(u(x, t))$ is measurable in $\Omega_{T}$.

As proved in [3], if $\left(u_{1}, u_{2}, s\right)$ is a classical solution of (1.1)-(1.5), and if we set

$$
\begin{aligned}
u & =u_{1} / \alpha_{1} \quad \text { at the points where } u_{1}>0, \\
& =u_{2} / \alpha_{2} \quad \text { at the points where } u_{2}<0,
\end{aligned}
$$

then $u$ is a weak solution of (1.1)-(1.5). Conversely, if $u$ is a weak solution, if the set where $u=0$ is given by a curve $x=s(t)$, and if $u$ and $s$ satisfy some smoothness conditions, then $\left(u_{1}, u_{2}, s\right)$ is a classical solution of (1.1)-(1.5), where the $u_{i}$ are defined by (1.10).

We shall need the following conditions:

$\left(\mathrm{A}_{1}\right) 0<\gamma_{1} \leqq g_{1}(t) \leqq \bar{\gamma}_{1}, \quad \bar{\gamma}_{2} \leqq g_{2} \leqq \gamma_{2}<0$.

$\left(\mathrm{A}_{2}\right)$ There exists a function $\Psi(x, t)$ with $\Psi_{x}, \Psi_{x x}, \Psi_{t}$ continuous in $\Omega_{T}$ such that $\Psi=g_{i}$ on $x=b_{i}$ and $\Psi=h_{i}$ on $t=0$, for all $x$ with $\left|x-b_{i}\right|$ sufficiently small.

$\left(\mathrm{A}_{3}\right) h$ is continuous on $G$ and its first strong derivative is in $L^{2}(G)$.

The following two theorems are contained in [3].

THEOREM 1. Assume that $\left(\mathrm{A}_{1}\right)-\left(\mathrm{A}_{3}\right)$ hold with some $T \leqq \infty$. Then there exists a unique weak solution $u(x, t)$ of (1.1)-(1.5). Furthermore, (i) $u(x, t)$ is a continuous function in $\bar{\Omega}_{T}$, and (ii) $u(x, t) \geqq \varepsilon_{0}$ for $b_{1} \leqq x \leqq b_{1}+\delta_{0}, 0 \leqq t<T$ and $u(x, t) \leqq-\varepsilon_{0}$ for $b_{2}-\delta_{0} \leqq x \leqq b_{2}, 0 \leqq t<T$, where $\varepsilon_{0}, \delta_{0}$ are positive constants.

THEOREM 2. Assume that $\left(\mathrm{A}_{1}\right)-\left(\mathrm{A}_{3}\right)$ hold with $T=\infty$ and that

$$
\left|g^{\prime}(t)\right| \leqq \text { const }<\infty, \quad \int^{\infty}\left|g^{\prime}(t)\right| d t<\infty, \quad \int^{\infty}(g(t)-g(\infty))^{2} d t<\infty
$$

Denote by $g_{i}(\infty) / \alpha_{i}$ the value of $g(\infty)$ on $x=b_{i}$, and by $w(x)$ the linear function in $\bar{G}$ which satisfies: $w\left(b_{i}\right)=g_{i}(\infty) / \alpha_{i}$. Then

$$
\sup _{x \in G}|u(x, t)-w(x)| \rightarrow 0 \quad \text { as. } t \rightarrow \infty
$$

Note that if the condition $\left(\mathrm{A}_{2}\right)$ holds with $T=\varepsilon$ for some $\varepsilon>0$ and if $g^{\prime}(t)$ is a continuous function for $0<t \leqq T_{0}$, then $\left(\mathrm{A}_{2}\right)$ holds also with $T=T_{0}$.

Set $W_{\sigma}=\{x \in G ; u(x, \sigma)=0\}, W=\bigcup_{0 \leqq \sigma} W_{\sigma}$. By [3], $u$ is a classical solution of the appropriate parabolic equation in the open set $\Omega_{T}-W$. We now state one of the principal results of the paper.

THEOREM 3. Assume that $\left(\mathrm{A}_{1}\right)-\left(\mathrm{A}_{3}\right)$ hold with some $T \leqq \infty$. Then the set $W$ coincides with a set $\{(x, t) ; x=s(t), 0 \leqq t<T\}$ where $s(t)$ is a continuous function for $0 \leqq t<T$.

Thus there is a free boundary in the usual sense. $u$ is a classical solution of (1.1) for $x \neq s(t)$, it satisfies (1.2)-(1.4) in the usual sense. The only condition which is satisfied in the weak sense is (1.5). This condition reads as follows: 
For every $0<t_{0}<T$ and $\phi \in C^{\infty}$ with compact support in a neighborhood of $\left(s\left(t_{0}\right), t_{0}\right)$ contained in $\Omega_{T}$,

$$
\begin{aligned}
\lim _{\varepsilon \rightarrow 0} \int \phi\left[\beta_{1} \partial u_{1} / \partial x\right]_{x} & =s_{\varepsilon}(t) \\
& =\lim _{\varepsilon \rightarrow 0} \iint_{\Omega_{T}^{\varepsilon}}\left[a(u) \phi_{t}+u_{x x} \phi\right] d x d t ;
\end{aligned}
$$

here $x=s_{\varepsilon}(t)$ and $x=s^{\varepsilon}(t)$ are smooth curves which approximate $x=s(t)$ uniformly, $s_{\varepsilon}(t)<s(t)<s^{\varepsilon}(t)$, and $\Omega_{T}^{\varepsilon}$ is the complement in $\Omega_{T}$ of the set of all points $(x, t)$ with $s_{\varepsilon}(t) \leqq x \leqq s^{\varepsilon}(t)$. If, in particular, $d s / d t$ exists and is continuous in some interval $\left|t-t_{0}\right|<\varepsilon^{\prime}$ and if $\partial u_{i}(x, t) / \partial x$ are continuous up to the free boundary for $\left|t-t_{0}\right|<\varepsilon^{\prime}$, then (as is easily seen from (1.13)) (1.5) is satisfied in the usual sense.

In $\$ 4$ we shall derive another weak form of (1.5), which is more explicit than (1.13).

From Theorems 2, 3 we get

COROLlaRY. Under the assumptions of Theorem 2,

$$
s(t) \rightarrow b_{1}+\alpha_{2} g_{1}(\infty)\left(b_{2}-b_{1}\right) /\left(\alpha_{2} g_{1}(\infty)-\alpha_{1} g_{2}(\infty)\right) \quad \text { as } t \rightarrow \infty .
$$

We shall need later on some additional results of [3].

Suppose $h(x)$ is any bounded measurable function on $\bar{G}$, and let $\beta(x)$ be a measurable function defined as $a(h(x))$ if $h(x) \neq 0$ and satisfying $-\alpha \leqq \beta(x) \leqq 0$ at points where $h(x)=0$. We shall write $a(h)$ (or $a(h(x))$ ) for the function $\beta$ (or $\beta(x)$ ).-We then define a weak solution of (1.1)-(1.5) with $a(u)=a(h)$ on $t=0$ by the condition (1.9). As an illustration we mention that Corollary 1 to Theorem 3 in [3] in fact asserts that the weak solution of $(1.1)-(1.5)$ is also, for $\sigma \leqq t<T$, a weak solution with $a(u)=a(k)$ on $t=\sigma$, where $k(x)=u(x, \sigma)$. Note that $a(h)$ actually determines $h$ uniquely.

THEOREM 4. If $g, h, a(h)$ are bounded measurable functions, then there exists $a$ unique weak solution of $(1.1)-(1.5)$ with $a(u)=a(h)$ on $t=0$.

COROLlARY. If $g^{*}, h^{*}, a\left(h^{*}\right)$ are also bounded measurable functions, and if $g^{*} \geqq g, a\left(h^{*}\right) \geqq a(h)$, then $u^{*} \geqq u$ almost everywhere, where $u^{*}$ is the weak solution with data $g^{*}, a\left(h^{*}\right)$.

The proofs of Theorem 4 and its corollary, for any $n \geqq 1$, are given in [3].

\section{Proof of Theorem 3.}

LEMMA 1. Let $D$ be a subdomain of $\Omega_{T}$ bounded by 4 curves: an interval $\gamma_{0}$ lying on $t=\mu_{0}$, an interval $\gamma_{1}$ lying on $t=\mu_{1}\left(\mu_{0}<\mu_{1}\right)$, a continuously differentiable closed curve $\gamma_{2}$ connecting the right end-points of $\gamma_{0}, \gamma_{1}$ and lying (except for its end-points) in $\mu_{0}<t<\mu_{1}$, and a continuously differentiable closed curve $\gamma_{3}$ connecting the left end-points of $\gamma_{0}, \gamma_{1}$ and lying (except for its end-points) in $\mu_{0}<t<\mu_{1}$. Assume that 
the tangent lines to $\gamma_{2}, \gamma_{3}$ are never parallel to the $x$-axis. If $u(x, t)>0$ on $\gamma_{0} \cup \gamma_{2} \cup \gamma_{3}$ then $u(x, t)>0$ in $\bar{D}$.

Proof. Since $u$ is continuous in $\bar{\Omega}_{T}$, the set $W$ where $u=0$ is a closed set. Hence, if the assertion is false then there exists a number $\sigma, \mu_{0}<\sigma \leqq \mu_{1}$, such that $u(x, t)>0$ in $D_{\sigma} \equiv D \cap\{t ; t<\sigma\}$ and $u\left(x^{0}, \sigma\right)=0$ for some point $\left(x^{0}, \sigma\right) \in \bar{D}_{\sigma}$ which does not lie on $\gamma_{2} \cup \gamma_{3}$. In $D_{\sigma}$ we have $a(u(x, t))=\alpha_{1} u(x, t)$ and thus, by $(1.9), u(x, t)$ is a weak (and, consequently, a classical) solution of $\alpha_{1} u_{t}=u_{x x}$. Consider next the solution $w$ of

$$
\begin{aligned}
\alpha_{1} w_{t} & =w_{x x} & & \text { in } D, \\
w & =u & & \text { on } \gamma_{0} \cup \gamma_{2} \cup \gamma_{3} .
\end{aligned}
$$

Its existence follows by [2]. By the maximum principle, $w>0$ in $\bar{D}$. By uniqueness, $w=u$ in $D_{\sigma}$. Since $u$ is continuous in $\bar{D}_{\sigma}$, we get $u\left(x^{0}, \sigma\right)=w\left(x^{0}, \sigma\right)>0$; a contradiction.

We shall need later on the following slightly stronger statement than Lemma 1.

LEMMA 1'. If in Lemma 1 the assumption that $u>0$ at the points $\bar{\gamma}_{1} \cap \gamma_{2}, \bar{\gamma}_{1} \cap \gamma_{3}$ is replaced by the assumption that $u \geqq 0$ at these points, then $u>0$ in $\bar{D}$ with the exception of these two points.

The only difference in the proof occurs in case $\sigma=\mu_{1}$. We can still proceed as before, but we have to apply to $w$, in $\bar{D}_{\mu_{1}}$, the strong maximum principle.

Set $B_{\sigma}=\left\{(x, \sigma) \in \bar{\Omega}_{T} ; u(x, \sigma)>0\right\}, B=\bigcup_{0 \leqq \sigma<T} B_{\sigma}$.

LEMMA 2. For each $\sigma, 0<\sigma<T$, the set $B_{\sigma}$ consists of one open interval.

Proof. Since $u$ is continuous in $\bar{\Omega}_{T}, B_{\sigma}$ is an open subset of $\bar{G} \times\{\sigma\}$. By Theorem $1, B_{\sigma}$ contains an interval with $b_{1} \leqq x \leqq b_{1}+\delta_{0}$ and excludes an interval with $b_{2}-\delta_{0} \leqq x \leqq b_{2}$. Suppose now that the assertion of the lemma is false. Then for some $\sigma$ with $0<\sigma<T$, the set $B_{\sigma}$ consist of disjoint (open) intervals $I_{0}, I_{1}, \ldots$ (whose number is at least 2) such that:

In $I_{0}, b_{1} \leqq x<c_{1}$.

In $I_{k}, c_{2 k}<x<c_{2 k+1}(k \geqq 1) ; c_{j} \nearrow$ if $j \nearrow$, and $c_{j}<b_{2}-\delta_{0}$ for all $j$. Note that $u\left(c_{j}, \sigma\right)=0$ for all $j$.

Consider open connected subsets $Q_{1}^{*}$ of $B$ lying in $\Omega_{\sigma}$ and having the following property: Each point $\left(x^{0}, t^{0}\right)$ of $Q_{1}^{*}$ can be connected to some point $(y, \sigma)$ of $I_{1}$ by a continuously differentiable curve $\gamma$ lying in $Q_{1}^{*}$ except for one end-point and given by $x=x(t)$ where $d x(t) / d t \neq 0$ for all $t$. We shall call such curves $\gamma$ monotone curves. The union $Q_{1}$ of all the sets $Q_{1}^{*}$ is again a set having the same property. Thus $Q_{1}$ is the maximal $Q_{1}^{*}$-set.

Let $\left(x^{0}, t^{0}\right)$ be a boundary point of $Q_{1}$ which does not belong to $\bar{I}_{1}$. Then $u\left(x^{0}, t^{0}\right) \geqq 0$. We shall prove

(i) If $u\left(x^{0}, t^{0}\right)>0$ then $0<t^{0}<\sigma$;

(ii) if $0<t^{0}<\sigma$ then $u\left(x^{0}, t^{0}\right)=0$.

It would then follow that $u(x, t)$ attains its maximum in $\bar{Q}_{1}$ on the set $Q_{1} \cup I_{1}$. 
Since $u_{1}$ is a solution of $\alpha_{1} u_{t}=u_{x x}$ in $Q_{1} \cup I_{1}$, the strong maximum principle yields $u \equiv$ const in $Q_{1}$, which is impossible. It thus remains to prove (i), (ii).

Suppose $t^{0}=\sigma$. Since $u\left(x^{0}, \sigma\right)>0$, there exists a disc $V$ with center $\left(x^{0}, \sigma\right)$ such that $u>0$ in $V$. Since $\left(x^{0}, \sigma\right) \in \bar{Q}_{1}$, we can find a point $\left(x^{\prime}, \sigma^{\prime}\right)$ lying in $Q_{1} \cap V$. Let $\gamma_{2}$ be a monotone curve in $Q_{1}$ connecting $\left(x^{\prime}, \sigma^{\prime}\right)$ to a point in $I_{1}$, and let $\gamma_{3}$ be the straight segment connecting $\left(x^{\prime}, \sigma^{\prime}\right)$ to $\left(x^{0}, \sigma\right)$. With $\gamma_{0}$ being the point $\left(x^{\prime}, \sigma^{\prime}\right)$, we have the situation considered in Lemma 1. It follows that $u(x, \sigma)>0$ for all $(x, \sigma)$ in the interval connecting $\left(x^{0}, \sigma\right)$ to some point of $I_{1}$. This is impossible since $u$ vanishes at the end-points of $I_{1}$.

Suppose next that $t^{0}=0$. Since $u\left(x^{0}, t^{0}\right)>0$, we must have $b_{1} \leqq x^{0}<b$. Take a disc $V$ about $\left(x^{0}, 0\right)$ such that $u>0$ in $V \cap \bar{\Omega}_{\sigma}$ and let $\left(x^{\prime}, \sigma^{\prime}\right)$ be a point lying in $V \cap \bar{\Omega}_{\sigma}$. Take $\gamma_{2}^{\prime}$ to be a monotone curve in $Q_{1}$ connecting $\left(x^{\prime}, \sigma^{\prime}\right)$ to some point in $I_{1}$. Connect $\left(x^{\prime}, \sigma^{\prime}\right)$ to $\left(x^{0}, 0\right)$ by a straight segment $\gamma_{2}^{\prime \prime}$ and set $\gamma_{2}=\gamma_{2}^{\prime} \cup \gamma_{2}^{\prime \prime}$. Denote by $\gamma_{0}$ the interval connecting $\left(x^{0}, 0\right)$ to $\left(b_{1}, 0\right)$ and by $\gamma_{3}$ the interval connecting $(b, 0)$ to $(b, \sigma)$. Then we can again apply Lemma 1 . We conclude that $u(x, \sigma)>0$ for all $x$ in the interval $b_{1} \leqq x \leqq c_{2}$. But this is impossible if $x=c_{2}$.

We now prove (ii). Suppose then that $u\left(x^{0}, t^{0}\right)>0$ and that $0<t^{0}<\sigma$. Let $V$ be a disc with center $\left(x^{0}, t^{0}\right)$, lying in $\Omega_{\sigma}$, such that $u>0$ in $V$. Denote by $V^{+}$and $V^{-}$ the intersection of $V$ with $t>t^{0}$ and $t<t^{0}$ respectively. Denote by $J_{\lambda \mu}$ the interval $\left\{\left(x, t^{0}\right) ; x^{0}-\lambda<x<x^{0}+\mu\right\}$. Since $\left(x^{0}, t^{0}\right)$ is not in $Q_{1}, V^{+} \cap Q_{1}=0$ (here we use the maximality of $\left.Q_{1}\right)$. Since $\left(x^{0}, t^{0}\right)$ is a boundary point of $Q_{1}$, there exists a sequence of points $\left(x_{m}, t_{m}\right)$ in $V^{-} \cap Q_{1}$ such that $x_{m} \rightarrow x^{0}, t_{m} \nearrow t^{0}$. It is therefore clear that $V^{-\subset} Q_{1}$.

Choose $\lambda, \mu$ such that $J_{\lambda \mu}$ is the largest interval on which $u\left(x, t^{0}\right)>0$. If the left end-point of $J_{\lambda \mu}$ lies on $x=b_{1}$, then we can construct a straight segment $\gamma_{0}$ lying on $t=t^{0}-\varepsilon$, for some sufficiently small $\varepsilon>0$, which begins on $x=b_{1}$ and ends at some point $P$ in $V^{-}$, such that $u>0$ on $\gamma_{0} . P$ can be connected to $I_{1}$ by a monotone curve $\gamma_{2}$ in $Q_{1}$. Denoting by $\gamma_{3}$ the interval connecting $\left(b, t^{0}-\varepsilon\right)$ to $(b, \sigma)$ we can now apply Lemma 1 and conclude that $u\left(c_{2}, \sigma\right)>0$, a contradiction.

We have thus proved that the left end-point of $J_{\lambda \mu}$ lies in $\Omega_{\sigma}$. The right end-point of $J_{\lambda \mu}$ also lies in $\Omega_{\sigma}$, since $u<0$ on $x=b_{2}$. Hence, by the maximality of $J_{\lambda \mu}, u=0$ at the end-points of $J_{\lambda \mu}$.

Let $\gamma^{\prime}$ be a monotone curve in $Q_{1}$ connecting $I_{1}$ to a point $\left(x^{\prime}, \sigma^{\prime}\right)$ in $V^{-} \cdot \gamma^{\prime}$ cannot intersect $J_{\lambda \mu}$ for, otherwise, we can construct a monotone curve connecting $I_{1}$ to $\left(x^{0}, t^{0}\right)$ on which $u>0$; thus $\left(x^{0}, t^{0}\right)$ would belong to $Q_{1}$, which contradicts the assumption that $\left(x^{0}, t^{0}\right)$ is a boundary point of $Q_{1}$. Thus, $\gamma^{\prime}$ intersects the line $t=t^{0}$ either to the right or to the left of $J_{\lambda \mu}$. Suppose it intersects it to the right of $J_{\lambda \mu}$, and denote by $\gamma_{2}$ the part of $\gamma^{\prime}$ lying in $t \leqq t^{0}$. We connect $\left(x^{\prime}, \sigma^{\prime}\right)$ to $\left(x^{0}, t^{0}\right)$ by a straight segment $\gamma_{3}$ and then apply Lemma 1 . We conclude that $u\left(x^{0}+\mu, t^{0}\right)>0$, a contradiction.

From Lemma 2 it follows that there is a curve $x=s_{+}(t)$ such that $u(x, t)>0$ if $b_{1} \leqq x<s_{+}(t), 0 \leqq t<T$, and $u(x, t) \leqq 0$ if $s_{+}(t)<x<b_{2}, 0 \leqq t<T$. Similarly one 
proves that there exists a curve $x=s_{-}(t)$ such that $u(x, t)<0$ if $s_{-}(t)<x \leqq b_{2}$, $0 \leqq t<T$ and $u(x, t) \geqq 0$ if $b_{1} \leqq x<s_{-}(t), 0 \leqq t<T$. Hence

$$
W_{\sigma}=\left\{(x, \sigma) ; s_{+}(\sigma) \leqq x \leqq s_{-}(\sigma)\right\} .
$$

The continuity of $u$ implies that $s_{+}(t)$ is a lower semicontinuous function and $s_{-}(t)$ is an upper semicontinuous function. If we prove that $s_{+}(t) \equiv s_{-}(t) \equiv s(t)$ then it would follow that the curve $x=s(t)$ is continuous and the proof of Theorem 3 is complete.

\section{Proof of Theorem 3 (continued).}

LEMMA 3. If $u=0$ on an interval

$$
I=\left\{(x, \sigma) ; x_{0} \leqq x \leqq x_{1}\right\}
$$

with $0<\sigma<T, x_{0}<x_{1}$, then there cannot exist any rectangle $R \equiv\left\{(x, t) ; y_{0} \leqq x \leqq y_{1}\right.$, $\sigma-\varepsilon \leqq t<\sigma\}$ where $x_{0} \leqq y_{0}<y_{1} \leqq x_{1}, \varepsilon>0$ such that $u>0$ in $R$.

Proof. The existence of such a rectangle contradicts Lemma 1'.

LEMMA 4. The function $t \rightarrow a(u(x, t))$ from $[0, T)$ into $L^{2}(G)$ is weakly continuous, i.e. for any $\phi \in L^{2}(G), 0 \leqq \sigma<T$,

$$
\lim _{t \rightarrow \sigma} \int_{G} a(u(x, t)) \phi(x) d x=\int_{G} a(u(x, \sigma)) \phi(x) d x .
$$

This lemma, which is true also when $x$ is $n$-dimensional, follows from Corollary 1 to Theorem 3 in [3].

REMARK. In the definition of a weak solution, $a(u(x, t))$ was defined up to a set of measure zero. In proving Lemma 4 one normalizes $a(u(x, t))$ in an appropriate manner so that the assertion holds for all $\sigma$. From now on this normalization is always assumed (also for the many-phase problems in \$§5-7 (Lemma 4 is valid also for such problems)). Thus, for each $\sigma, 0 \leqq \sigma<T$, the function $a(u(x, \sigma))$ is a uniquely defined measurable function in $x$, i.e. it is determined up to a set of measure zero in $G$.

Assume now that there is an interval $I$ as in (3.1) on which $u=0$. Suppose a point $(y, \sigma)$ in $I$ is a limit point of a sequence $\left\{\left(y_{m}, \sigma_{m}\right)\right\}$ with $\sigma_{m} \nearrow \sigma$ and that $u\left(y_{m}, \sigma_{m}\right)>0$ for all $m$. By Lemma $2, u\left(x, \sigma_{m}\right)>0$ if $b_{1} \leqq x \leqq y_{m}$ and, therefore, $a\left(u\left(x, \sigma_{m}\right)\right)$ $=\alpha_{1} u\left(x, \sigma_{m}\right)$. Since, by Lemma $4, a\left(u\left(x, \sigma_{m}\right)\right) \rightarrow a(u(x, \sigma))$ in $L^{2}{ }_{\left(b_{1}, y\right)}$ (where " $\rightarrow$ " means weak convergence), and since $u\left(x, \sigma_{m}\right) \rightarrow 0$ as $m \rightarrow \infty$, for all $x$ in the interval $x_{0}<x<y$, we conclude that $a(u(x, \sigma))=0$ for almost all $x$ in the interval $x_{0} \leqq x \leqq y$.

If, on the other hand, $u\left(y_{m}, \sigma_{m}\right)<0$ for all $m$, where $\left\{\left(y_{m}, \sigma_{m}\right)\right\}$ is a sequence which converges to $(y, \sigma) \in I$ as before, then we conclude in a similar manner tha $a(u(x, \sigma))=-\alpha$ for almost all $x$ in the interval $y \leqq x \leqq x_{1}$.

It follows that if $(y, \sigma) \in I$ is a limit of a sequence $\left\{\left(y_{m}, \sigma_{m}\right)\right\}$ with $u\left(y_{m}, \sigma_{m}\right)>0$, then each point $(\bar{y}, \sigma)$ with $x_{0} \leqq \bar{y}<y$ is not a limit point of a sequence of points on which $u<0$. On the other hand, each such point $(\bar{y}, \sigma)$ is a limit of the sequence $\left\{\left(\bar{y}, \sigma_{m}\right)\right\}$ on which $u>0$ (by Lemma 2 ). 
From the above remarks it follows that there exist points $\bar{x}^{0}, \bar{x}_{1}$ with $x_{0} \leqq \bar{x}_{0}$ $\leqq \bar{x}_{1} \leqq x_{1}$ such that the following properties hold:

( $\alpha$ If $x_{0} \leqq y<\bar{x}_{0}$, then there exists a sequence $\left\{\left(y_{m}, \sigma_{m}\right)\right\}$ such that $y_{m} \rightarrow y$, $\sigma_{m} \nearrow \sigma, u\left(y_{m}, \sigma_{m}\right)>0$ for all $m$.

$(\beta)$ If $\bar{x}_{1}<z \leqq x_{1}$ then there exists a sequence $\left\{\left(z_{m}, \sigma_{m}\right)\right\}$ such that $z_{m} \rightarrow z, \sigma_{m} \nearrow \sigma$, $u\left(z_{m}, \sigma_{m}\right)<0$ for all $m$.

$(\gamma) \bar{x}_{0}$ is the largest value and $\bar{x}_{1}$ is the smallest value for which $(\alpha),(\beta)$ hold.

There are three cases (the first two may occur simultaneously):

(3.2) $x_{0}<\bar{x}_{0}$;

(3.3) $\bar{x}_{1}<x_{1}$;

(3.4) $x_{0}=\bar{x}_{0}, \quad x_{1}=\bar{x}_{1}$.

Later on we shall analyze these cases.

Definitions. A closed interval (of positive length) on which $u=0$ will be called a zero interval. We shall consider below zero intervals $J_{y \mu}=\{(y, t) ; \sigma-\mu \leqq t \leqq \sigma\}$. If $J_{y \mu}$ is not contained in any zero interval $J_{y v}$ with $\nu<\mu$ then we call it maximal. If $J_{y \mu}$ is maximal and $\sigma-\mu>0$, then there exists a sequence of points $\left(y, \lambda_{m}\right)$ with $\lambda_{m} \nearrow \sigma-\mu$ such that $u\left(y, \lambda_{m}\right) \neq 0$ for all $m$. If one can find such a sequence with $u\left(y, \lambda_{m}\right)>0(<0)$ then one says that $J_{y \mu}$ has a positive (negative) end. $J_{y \mu}$ may have both a positive end and a negative end simultaneously.

LeMma 5. Suppose $u=0$ on an interval I given by (3.1). Denote by $Y$ the set of all $y$ with $x_{0}<y<x_{1}$ such that there exists a zero interval $J_{y \mu}$. Then the set $Y$ cannot contain any subinterval $\left(z_{0}, z_{1}\right)$ of $\left(x_{0}, x_{1}\right)$.

Proof. We may assume that the $J_{y \mu}$ are maximal zero intervals. We may also assume that $\mu<\sigma$. Indeed, there can be at most one value of $y$ (namely $y=b$ ) for which $\mu=\sigma($ since $u(x, 0) \neq 0$ if $x \neq b)$.

Assume now that the assertion is false, i.e. $Y$ contains an interval $\left(z_{0}, z_{1}\right)$.

There exist two points $y_{1}, y_{2}$ satisfying $z_{0}<y_{1}<y_{2}<z_{1}$ such that the intervals $J_{y_{1} \mu_{1}}$ and $J_{y_{2} \mu_{2}}$ (where $\mu_{i}=\mu\left(y_{i}\right)$ ) have both either a positive end or a negative end. It suffices to consider the case of a positive end. By Lemma 2 it follows that $\mu_{1} \leqq \mu_{2}$. Suppose $y_{1}<y<y_{2}$. If $\mu_{1}=\mu_{2}$ then, by Lemma $2, J_{y \mu}$ has a positive end and $\mu_{1} \leqq \mu \leqq \mu_{2}$. If $\mu_{1}<\mu_{2}$ then $J_{y \mu}$ also must have a positive end. In fact, if it has a negative end then Lemma 2 implies that $\mu=\mu_{2}$ and thus $J_{y \mu}$ must also have a positive end. From the positivity of the end of $J_{y \mu}$ and from Lemma 2 it now follows that $\mu_{1} \leqq \mu \leqq \mu_{2}$.

Dividing the interval $\left(y_{1}, y_{2}\right)$ successively into smaller intervals whoso lengths decrease to 0 , and applying successively the last result, we conclude that $\mu=\mu(y)$ is a monotone increasing function in the intervals $\left[y_{1}, y_{2}\right]$ and $J_{y \mu}$ has a positive end.

Denote by $\Lambda$ the curve whose points are $(\sigma-\mu(y), y), y_{1}<y<y_{2}$. Let $\bar{y}$ be a point in $\left(y_{1}, y_{2}\right)$, and set $t_{0}=\sigma-\bar{\mu}$ where $\bar{\mu}=\mu(\bar{y})$. Since $J_{\overline{0} \bar{u}}$ has a positive end, by applying Lemmas 2,4 we find that $a\left(u\left(x, t_{0}\right)\right)=\alpha_{1} u\left(x, t_{0}\right)$ for almost all $x$ in the interval $\left(b_{1}, \bar{y}\right)$. 
Denote by $\Omega^{*}$ the rectangle $b_{1}<x<\bar{y}+\varepsilon, t_{0}<t<\sigma$ for some $\varepsilon>0, \varepsilon<y_{2}-\bar{y}$. We also introduce the segment $S=\left\{(\bar{y}+\varepsilon, t) ; t_{0}<t<\sigma\right\}$. Clearly, $u$ vanishes in some neighborhood of $S$ which lies in $t_{0} \leqq t \leqq \sigma$. From this it easily follows that $u$, which (by Corollary 1 to Theorem 3 of [3]) is a weak solution of (1.1)-(1.5) in $G \times\left(t_{0}, \sigma\right)$ (with $a(u)=a(k)$ on $t=t_{0}$ where $k(x)=u\left(x, t_{0}\right)$ ) is also a weak solution in $\Omega^{*}$ of the appropriate free boundary problem.

Note that $a(k(x))=\alpha_{1} u(x, \sigma)>0$ if $b_{1}<x<\bar{y}$ and $-\alpha \leqq a(k(x)) \leqq 0$ if $\bar{y}<x<\bar{y}+\varepsilon$.

We shall compare $u$ with the solution $w$ of the free boundary problem with boundary data $g_{1}(t)$ on $x=b_{1}, 0$ on $x=\bar{y}+\varepsilon$, and initial data on $t=t_{0}$ such that $a(w) \leqq a(u)$. We take $w\left(x, t_{0}\right)=0$ for $b_{1}<x<\bar{y}+\varepsilon, a\left(w\left(x, t_{0}\right)\right)=0$ for $b_{1}<x<\bar{y}$ and $a\left(w\left(x, t_{0}\right)\right)=-\alpha$ for $\bar{y}<x<\bar{y}+\varepsilon$. By [1] there exists a classical solution $w, s$ with the free boundary satisfying $s\left(t_{0}\right)=\bar{y}$, and $s(t)$ is a monotone increasing function. Thus $w(x, t)>0$ if $b_{1}<x<\bar{y}, t_{0}<t<\sigma . w$ is also a weak solution with $a\left(w\left(x, t_{0}\right)\right)$ as prescribed above.

By the corollary to Theorem $4, u(x, t) \geqq w(x, t)>0$ if $b_{1}<x<\bar{y}, t_{0}<t<\sigma$. But this is impossible since $u=0$ on $J_{y_{1} \mu_{1}}$.

REMARK. Lemma 5 implies that the set in $\Omega_{T}$ where $u=0$ has no interior points. It is this fact (rather than the stronger assertion of Lemma 5) that will be used in the future.

LEMMA 6. Let I be a zero interval given by (3.1) and let $\bar{x}_{0}, \bar{x}_{1}$ be the values which occur in (3.2)-(3.4). Then $\bar{x}_{0}=\bar{x}_{1}$.

Indeed, if $\bar{x}_{0}<\bar{x}_{1}$ then there exists a rectangle $R_{0}$ given by $\bar{x}_{0}+\varepsilon \leqq x \leqq \bar{x}_{1}-\varepsilon$, $\sigma-\varepsilon \leqq t \leqq \sigma$ such that $u \equiv 0$ in $R_{0}$. This contradicts Lemma 5 .

We can now complete the proof of Theorem 3. From Lemma 6 it follows that either $\bar{x}_{0}>x_{0}$ or $\bar{x}_{1}<x_{1}$ (or both). It suffices to consider the case $\bar{x}_{0}>x_{0}$. Then there exists a rectangle $R$ given by $x_{0} \leqq x \leqq x_{0}+\delta, \sigma-\delta<t<\sigma$, for some $\delta>0$, such that $u \geqq 0$ in $R$. Let $\pi$ be any zero interval in $R$, lying on a line $t=$ const $>\sigma-\delta$. Applying Lemma 6 to $\pi$ and recalling that $u \geqq 0$ in $R$, we conclude that every point in $\pi$ is the limit of a sequence in $R$ on which $u>0$. By Lemmas 2, 4 it follows that $a(u)=\alpha_{1} u$ almost everywhere on $\pi$. Consequently $a(u)=\alpha_{1} u$ almost everywhere in $R$. From (1.9) we then conclude that $u$ is a weak (and hence a classical) solution of $\alpha_{1} u_{t}=u_{x x}$ in $R$. Since $u \geqq 0$ and $u \neq 0$ in $R$, the maximum principle shows that $u(x, t)>0$ in $R$. But this contradicts Lemma 3 .

4. Another weak form of the condition on the free boundary. Recalling the notation (1.10), we shall prove the following theorem.

THEOREM 5. Assume that $\left(\mathrm{A}_{1}\right)-\left(\mathrm{A}_{3}\right)$ hold for some $T \leqq \infty$. Then

$$
\begin{aligned}
\alpha s(t)= & \alpha s(0)-\int_{b_{1}}^{s(t)} u_{1}(x, t) d x-\int_{s(t)}^{b_{2}} u_{2}(x, t) d x+\int_{b_{1}}^{b} h_{1}(x) d x \\
& +\int_{b}^{b_{2}} h_{2}(x) d x+\beta_{2} \int_{0}^{t} \frac{\partial}{\partial x} u_{2}\left(b_{2}, \sigma\right) d \sigma-\beta_{1} \int_{0}^{t} \frac{\partial}{\partial x} u_{1}\left(b_{1}, \sigma\right) d \sigma .
\end{aligned}
$$


The relation (4.1) may be considered as another weak form of (1.5). In fact, suppose $u_{i}, \partial u_{i} / \partial x, \partial^{2} u_{i} / \partial x^{2}$ exist and are continuous for $b_{1} \leqq x \leqq s(t),\left|t-t_{0}\right|<\varepsilon$ if $i=1$ and for $s(t) \leqq x \leqq b_{2},\left|t-t_{0}\right|<\varepsilon$ if $i=2$. Suppose also that $d s(t) / d t$ exists and is continuous if $\left|t-t_{0}\right|<\varepsilon$. Then, writing (4.1) for $t=t_{0}+\delta$ and for $t=t_{0}$, dividing the difference by $\delta$ and taking $\delta \rightarrow 0$, we obtain the relation (1.5).

Proof of Theorem 5. In [3] we have considered the system

$$
\begin{aligned}
a_{m}^{\prime}\left(v_{m}\right) \partial v_{m} / \partial t & =\partial^{2} v_{m} / \partial x^{2} \text { in } \Omega_{T}, \\
v_{m} & =g \quad \text { on } x=b_{1}, x=b_{2}, 0<t<T, \\
v_{m} & =h \text { for } x \in G, t=0,
\end{aligned}
$$

and proved that some subsequence of $\left\{v_{m}\right\}$ is convergent weakly in $L^{2}\left(\Omega_{T}\right)$ and almost everywhere in $\Omega_{T}$ to a function $u$ which is the weak solution of (1.1)-(1.5). Furthermore, the convergence is uniform for $(x, t) \in \Omega_{\delta_{0}}^{*}$ where $\Omega_{\delta_{0}}^{*}$ is defined by

$$
b_{1} \leqq x \leqq b_{1}+\delta_{0} \quad \text { or } \quad b_{2}-\delta_{0} \leqq x \leqq b_{2}, \quad 0 \leqq t<T
$$

Finally, we have also proved that

$$
\sup _{0<t<T} \int\left|\frac{\partial}{\partial x} v_{m}(x, t)\right|^{2} d x \leqq C
$$

where $C$ is independent of $m$.

From Sobolev's lemma and (4.5) we find that

$$
\left|v_{m}(x, t)-v_{m}\left(x^{\prime}, t\right)\right| \leqq C^{\prime}\left|x-x^{\prime}\right|^{1 / 2} \quad\left(x, x^{\prime} \in G, 0<t<T\right),
$$

where $C^{\prime}$ is a constant independent of $m$.

From the differentiality assumptions $\left(\mathbf{A}_{2}\right),\left(\mathbf{A}_{3}\right)$ and the fact (also proved in [3]) that in $\Omega_{\delta_{0}}^{*} a_{m}^{\prime}\left(v_{m}\right)=\alpha_{1}$ if $b_{1} \leqq x \leqq b_{1}+\delta_{0}$ and $a_{m}^{\prime}\left(v_{m}\right)=\alpha_{2}$ if $b_{2}-\delta_{0} \leqq x \leqq b_{2}$, it follows that for a suitable subsequence of the $m$ 's.

$$
\partial v_{m} / \partial x \rightarrow \partial u / \partial x \quad \text { uniformly in } \Omega_{\delta}^{*}
$$

for any $0<\delta<\delta_{0}$.

Integrating (4.2) over $\Omega_{t}$ we obtain

$$
\int_{0}^{t}\left[\frac{\partial v_{m}(b, \sigma)}{\partial x}-\frac{\partial v_{m}(b, \sigma)}{\partial x}\right] d \sigma=\int_{b_{1}}^{b_{2}} a_{m}\left(v_{m}(x, t)\right) d x-\int_{b_{1}}^{b_{2}} a_{m}(h(x)) d x
$$

For fixed $t$ we can take (because of (4.6)) a subsequence of $\left\{v_{m}\right\}$ such that $v_{m}(x, t) \rightarrow v(x, t)$ uniformly with respect to $x$. Using Theorem 3 , we find

$$
\int_{b_{1}}^{b_{2}} a_{m}\left(v_{m}(x, t)\right) d x \rightarrow \int_{b_{1}}^{s(t)} \alpha_{1} u(x, t) d x+\int_{s(t)}^{b_{2}} \alpha_{2} u(x, t) d x-\int_{s(t)}^{b_{2}} \alpha d x .
$$


Taking in (4.8) $m \rightarrow \infty$ on an appropriate subsequence of $m$ 's, and using (4.9), (4.7) and the notation of (1.8), (1.10), we get (4.1).

5. The many-phase problem. We shall generalize the previous results to the case of many-phase problems. Thus, on $t=0$ there are given $k$ intervals $\left(d_{1}, d_{2}\right)$, $\left(d_{2}, d_{3}\right), \ldots,\left(d_{k}, d_{k+1}\right)$ with $d_{1}=b_{1}, d_{k+1}=b_{2}$.

On each interval $\left(d_{i}, d_{i+1}\right)$ we are given a function $h_{i}(x)$, and $(-1)^{i-1} h_{i}(\dot{x})>0$. In the classical formulation of the problem we are looking for $k$ functions $u_{1}, \ldots, u_{k}$ and $k-1$ free boundaries $s_{1}, \ldots, s_{k-1}$ such that $b_{1}<s_{1}(t)<s_{2}(t)<\cdots<s_{k-1}(t)<b_{2}$ and $u_{i}$ is defined for $s_{i-1}(t)<x<s_{i}(t)$ (here $\left.s_{0} \equiv b_{1}, s_{k} \equiv b_{2}\right)$. $u_{i}$ satisfies $u_{t}=\beta_{1} u_{x x}$ if $i$ is odd and $u_{t}=\beta_{2} u_{x x}$ if $i$ is even. $u_{i}=0$ on the free boundaries which bound its domain of definition, $u_{i}=h_{i}$ for $t=0, d_{i}<x<d_{i+1}, u_{1}=g_{1}$ for $x=b_{1}, 0<t<T$, $u_{k}=g_{2}$ for $x=b_{2}, 0<t<T$, and, finally,

$$
\beta_{1} \frac{\partial u_{i}}{\partial x}-\beta_{2} \frac{\partial u_{i+1}}{\partial x}=(-1)^{i} \alpha \frac{d s_{i}}{d t} \quad \text { on } x=s_{t}(t) \text { for } i=1, \ldots, k-1
$$

Actually, some of the phases may disappear after some time $t$, so that the classical formulation needs to be modified. We shall not give here such a modification, but instead proceed immediately to the weak formulation of the problem.

The notations (1.6), (1.7) will be kept, but instead of (1.8), (1.10) we set

$$
\begin{aligned}
h & =h_{i} / \alpha_{1} & & \text { for } d_{i}<x<d_{i+1}, i \text { odd, } \\
& =h_{i} / \alpha_{2} & & \text { for } d_{i}<x<d_{i+1}, i \text { even } \\
& =0 & & \text { for } x=d_{i}, \\
& & & \\
u & =u_{i} / \alpha_{1} & & \text { if } u_{i}>0, \\
& =u_{i} / \alpha_{2} & & \text { if } u_{i}<0 .
\end{aligned}
$$

Definition. A bounded measurable function $u$ in $\Omega_{T}$ is called a weak solution of the $k$-phase Stefan problem (with initial data $h$ and boundary data $g$ ) if (1.9) holds for all $\phi$ such that $\phi_{x}, \phi_{x x}, \phi_{t}$ are continuous in $\bar{\Omega}_{T}$ and $\phi=0$ on $x=b_{1}$, $x=b_{2}$ and on $t=T$. The meaning of $a(u)$ is the same as in $\S 1$.

One can show that a classical solution is a weak solution (via the relation (5.2)).

Theorems 1, 2, 4 remain true for the $k$-phase problem, and only slight changes in the original proofs are required. Thus, in proving the existence of a region $\Omega_{i}^{*}$ as in (2.10) of [3], which is independent of $m$, one constructs the function $w$ satisfying $w<h$ and (2.9) (of [3]) simply as a linear function. Theorems 1, 2, 4 obviously extend also to the case where $g_{2}(t)>0$, i.e. the case where $k$ is odd.

Our main purpose is to generalize Theorem 3. From now on it is always assumed that the assumptions $\left(\mathrm{A}_{1}\right)-\left(\mathrm{A}_{3}\right)$ (if $k$ is odd then $\left.0<\gamma_{2} \leqq g_{2}(t) \leqq \bar{\gamma}_{2}\right)$ hold.

Lemmas 1, 1', 3, 4 remain true (with the same proofs) for the $k$-phase problem.

On $t=\sigma$, the set where $u(x, \sigma)>0$ consists of disjoint intervals $I_{1}, I_{2}, \ldots$ They are all open intervals except the one initiating at $x=b_{1}$ (and the one ending on 
$x=b_{2}$ in case $g_{2}(t)>0$, i.e. in case $k$ is odd). We call the $I_{j}$ positive phases and denote their number by $n_{+}(\sigma)$. Similarly we define negative phases and denote their number by $n_{-}(\sigma)$.

THEOREM 6. If $\sigma>\sigma^{\prime}$ then $n_{+}(\sigma) \leqq n_{+}\left(\sigma^{\prime}\right), n_{-}(\sigma) \leqq n_{-}\left(\sigma^{\prime}\right)$.

Proof. It suffices to prove the assertion for $n_{+}$. Set $m=n_{+}(\sigma), m^{\prime}=n_{+}\left(\sigma^{\prime}\right)$ and denote by $I_{1}, \ldots, I_{m}$ and $I_{1}^{\prime}, \ldots, I_{m}^{\prime}$ the positive phases on $t=\sigma$ and $t=\sigma^{\prime}$ respectively. We enumerate these intervals in such a way that $I_{i}\left(I_{i}^{\prime}\right)$ is to the left of $I_{j}$ $\left(I_{j}^{\prime}\right)$ if $i<j$. In $\$ 2$ we have defined the domain $Q_{1}$ with respect to $I_{1}$. We now introduce in the same manner the domain $Q_{i}$ with respect to $I_{i}$, and set $\hat{Q}_{i}=Q_{i} \cap\left\{t>\sigma^{\prime}\right\}$.

Let $\left(x^{0}, t^{0}\right)$ be a boundary point of $\hat{Q}_{i}$ which does not belong to $\bar{I}_{i}$. We claim

(i) If $u\left(x^{0}, t^{0}\right)>0$ then $t^{0}<\sigma$; if $u\left(x^{0}, t^{0}\right)>0$ and $t^{0}=\sigma^{\prime}$ then $\left(x^{0}, t^{0}\right)$ belongs to some interval $I_{j(i)}^{\prime}$ and $j(i) \neq j(i)$ if $i \neq i$.

(ii) If $\sigma^{\prime}<t^{0}<\sigma$, then $u\left(x^{0}, t^{0}\right)=0$.

The proofs of (i), (ii) are similar to the proofs of the statements (i), (ii) which occur in the proof of Lemma 2.

If now $m>m^{\prime}$, then it follows from (i), (ii) that, for some $i, u=0$ on all the boundary of $\hat{Q}_{i}$ with the exception of $I_{i}$ where $u>0$. But this contradicts the maximum principle for $u$ in $\hat{Q}_{i}$.

From Theorem 6 and the continuity of $u$ we get

COROllaRY. $n_{+}(\sigma)$ and $n_{-}(\sigma)$ are continuous at $\sigma=0$; thus there exists $a \sigma_{1}>0$ such that $n_{+}(\sigma)=n_{+}(0), n_{-}(\sigma)=n_{-}(0)$ if $0 \leqq \sigma<\sigma_{1}$.

We shall take $\sigma_{1}$ to be the largest number such that $n_{+}(\sigma), n_{-}(\sigma)$ are constants in the interval $\left[0, \sigma_{1}\right)$.

LEMMA 7. There are no zero intervals of the form (3.1) for any $0<\sigma<\sigma_{1}$.

Proof. We use the notation $Q_{\mathfrak{i}}$ introduced above. Then (i), (ii) hold with $\sigma^{\prime}=0$, i.e. with $\hat{Q}_{i}=Q_{i}$. Using Lemma 1 we immediately see that $Q_{i} \cap Q_{i}=\varnothing$ if $i \neq j$.

Each $Q_{i}$ must have a boundary point on $t=0$ where $u>0$, since, otherwise, by (i), (ii) and the maximum principle, $u \equiv 0$ in $Q_{i}$ which is impossible. That boundary point must belong to some interval $\left(d_{j(i)}, d_{j(i)+1}\right)$. It is now easily seen that there exists a monotone curve $\gamma_{i}$ in $Q_{i}$ connecting $I_{i}$ to some point in $\left(d_{j(i)}, d_{j(i)+1}\right)$. Since the $I_{i}$ are arranged in a monotone increasing order on $t=\sigma$, and since $j(i) \neq j(i)$ if $i \neq i$, the interval $\left(d_{f(i)}, d_{j(i)+1}\right)$ is actually the interval $\left(d_{2 i-1}, d_{2 i}\right)$. Note that $\gamma_{i} \cap \gamma_{m}=\varnothing$ if $i \neq m$.

In the same way one proves that there exist monotone curves $\tilde{\gamma}_{i}$ connecting the negative phases $J_{i}$ on $t=\sigma$ to the intervals $\left(d_{2 i}, d_{2 i+1}\right)$, such that $u<0$ on $\tilde{\gamma}_{i}$, and $\tilde{\gamma}_{i} \cap \tilde{\gamma}_{m}=\varnothing$ if $i \neq m$. 
$\gamma_{i}$ can be represented in the form $x=x_{i}(\rho), 0 \leqq \rho \leqq \sigma$. Similarly, $\tilde{\gamma}_{i}$ can be represented in the form $x=\tilde{x}_{i}(\rho), 0 \leqq \rho \leqq \sigma$. Hence there exist intervals $I_{i}(\rho)=\left(x_{i}(\rho)-\varepsilon_{i}(\rho)\right.$, $\left.x_{i}(\rho)+\delta_{i}(\rho)\right)$ and $J_{i}(\rho)=\left(\tilde{x}_{i}(\rho)-\tilde{\varepsilon}_{i}(\rho), \tilde{x}_{i}(\rho)+\tilde{\delta}_{i}(\rho)\right)$ on $t=\rho$ such that $u(x, \rho)>0$ on $I_{i}(\rho), u(x, \rho)<0$ on $J_{i}(\rho)$ and $u=0$ at the end-points of these intervals. Since $n_{+}(\rho)=n_{+}(\sigma), n_{-}(\rho)=n_{-}(\sigma)$, we actually have

$$
u(x, \rho)=0 \text { if } x \notin\left(\bigcup_{i} I_{i}(\rho)\right) \cup\left(\bigcup_{i} J_{i}(\rho)\right)
$$

Suppose now that the Lemma is false. Then there exists a zero interval $I$ of the form (3.1). It lies between two intervals $I_{i}, J_{i}$ or $J_{i}, I_{i+1}$. It will be enough to consider the first case.

Denote by $\Omega_{i}$ the region bounded by $\gamma_{i}, \tilde{\gamma}_{i}, t=0$ and $t=\sigma$. If we can extend Lemma 5 to the case of zero intervals of the form (3.1) lying in $\Omega_{i}$, then we can derive a contradiction to the fact that $I$ (on $\Omega_{i} \cap\{t=\sigma\}$ ) is a zero interval using the same arguments as at the end of $\S 3$.

Note that zero intervals of the form $J_{y \mu}$ cannot intersect $\gamma_{i} \cup \tilde{\gamma}_{i}$ and thus must stay in the interior of $\Omega_{i}$ (except for their initial point on $I$ ). Following the proof of Lemma 5 we shall construct a weak solution $w$ to a free boundary problem in $\Omega^{*} \cap\left\{t_{0}<t<t_{0}+\delta\right\}$, for some $\delta<\sigma-t_{0}$, with boundary data $\leqq g_{1}(t)$ on $x=b_{1}$, 0 on $x=\bar{y}+\varepsilon$ and with $a(w) \leqq a(u)$ on $t=t_{0}$ such that $w(x, t)>0$ on the points $(y, t)$ with $y_{1}<y<\bar{y}, \sigma-\mu(y)<t<\sigma$ which lie sufficiently close to $\left(\bar{y}, t_{0}\right)$. (Such points exist if we choose $\left(\bar{y}, t_{0}\right)$ to be a point of continuity of $\Lambda$.) Since by the corollary to Theorem $4, u \geqq w$ in $\Omega^{*} \cap\left\{t_{0}<t<t_{0}+\delta\right\}$ and since $u(y, t)=0$ if $y_{1}<y<\bar{y}, \sigma-\mu(y) \leqq t \leqq \sigma$, we will thus get a contradiction.

Since $u>0$ on $\gamma_{i}$, there exist points on the interval $\left\{\left(x, t_{0}\right) ; \bar{x}<x<\bar{y}\right\}$, where $\bar{x}=\gamma_{1} \cap\left\{t=t_{0}\right\}$, on which $u>0$. We therefore can take $w\left(x, t_{0}\right)$ to be a continuously differentiable function for $b_{1} \leqq x \leqq \bar{y}+\varepsilon$, satisfying $w\left(x, t_{0}\right)<0$ if $b_{1}<x<\bar{x}$, $w\left(x, t_{0}\right) \geqq 0$ and $\not \equiv 0$ if $\bar{x}<x<\bar{y}, w\left(x, t_{0}\right)=0$ if $\bar{y}<x<\bar{y}+\varepsilon, w\left(x, t_{0}\right) \leqq u\left(x, t_{0}\right)$ if $b_{1}<x<\bar{y}+\varepsilon$. We take $a\left(w\left(x, t_{0}\right)\right)=0$ if $w\left(x, t_{0}\right)=0, \bar{x}<x<\bar{y}$, and $a\left(w\left(x, t_{0}\right)\right)=-\alpha$ if $\bar{y}<x<\bar{y}+\varepsilon$. We also take the boundary data on $x=b_{1}$ to be negative and smooth and on $x=\bar{y}+\varepsilon$ to be 0 .

By the methods of [1], there exists a classical solution $\left(w, s_{1}, s_{2}\right)$ in some interval $t_{0}<t<t_{0}+\delta$, where $s_{1}(t), s_{2}(t)$ are the two free boundaries, and $s_{1}\left(t_{0}\right)=\bar{x}, s_{2}(t) \equiv \bar{y}$. Using the maximum principle we find that $w(x, t)>0$ if $t_{0}<t<t_{0}+\delta, s_{1}(t)<x<s_{2}(t)$. Hence $w(x, t)>0$ on the points $(y, t)$ with $y_{1}<y<\bar{y}, t_{0}<t<t_{0}+\delta$ which are sufficiently close to $\left(y, t_{0}\right)$. Since $w$ is also a weak solution, the desired contradiction is obtained.

We have thus completed the proof of Lemma 7.

From Lemma 7 and the continuity of $u$ it follows that there exist $k-1$ continuous curves $x=s_{i}(t)$ for $0<t<\sigma_{1}$ such that $b_{1}<s_{1}(t)<s_{2}(t)<\cdots<s_{k-1}(t)<b_{2}$; $u(x, t)>0$ if $s_{2 t}(t)<x<s_{2 i+1}(t)$ and $u(x, t)<0$ if $s_{2 j-1}(t)<x<s_{2 j}(t)$. (Here $s_{0}(t) \equiv b_{1}, s_{k}(t) \equiv b_{2}$.)

Naturally, we call these curves the free boundaries of the $k$-phase problem. 
THEOREM 7. There exist $k-1$ continuous functions $s_{i}(t)$, for $0 \leqq t<\sigma_{1}$, such that $b_{1}<s_{1}(t)<\cdots<s_{k-1}(t)<b_{2}$ and such that $u(x, t)>0$ if $s_{2 i}(t)<x<s_{2 i+1}(t), 0 \leqq t<\sigma_{1}$ and $u(x, t)<0$ if $s_{2 i-1}(t)<x<s_{2 i}(t), 0 \leqq t<\sigma_{1}$ (here $\left.s_{0}(t) \equiv b_{1}, s_{k}(t) \equiv b_{2}\right)$. Furthermore,

$$
\begin{aligned}
\alpha \sum_{i=1}^{k-1}(-1)^{i-1} s_{i}(t)= & \alpha \sum_{i=1}^{k-1}(-1)^{i-1} s_{i}(0)-\sum_{i=1}^{k-1} \int_{s_{i}(t)}^{s_{i+1}(t)} u_{i}(x, t) d x \\
& +\sum_{i=0}^{k-1} \int_{s_{i}(0)}^{s_{i+1}(0)} h_{i}(x) d x+\beta_{2} \int_{0}^{t} \frac{\partial}{\partial x} u_{2}\left(b_{2}, \sigma\right) d \sigma \\
& -\beta_{1} \int_{0}^{t} \frac{\partial}{\partial x} u_{1}\left(b_{1}, \sigma\right) d \sigma .
\end{aligned}
$$

We have already proved all the assertions of the theorem except for (5.3). The proof of (5.3) is similar to the proof of Theorem 5 and is therefore omitted.

6. Degeneracy of phases of order 2. We denote by $I_{i}(\sigma)(0<\sigma<T)$ that positive phase on $t=\sigma$ which can be joined to the interval $\left(d_{2 i-1}, d_{2 i}\right)$ on $t=0$ by a monotone curve along which $u>0$. Similarly we denote by $J_{j}(\sigma)$ that negative phase on $t=\sigma$ which can be joined to the interval $\left(d_{2 i}, d_{2 i+1}\right)$ on $t=0$ by a monotone curve along which $u<0$. These notations agree with notations introduced in $\S 5$ for $0 \leqq \sigma<\sigma_{1}$.

Set $k(\sigma)=n_{+}(\sigma)+n_{-}(\sigma)$. If $k\left(\sigma_{1}-0\right)-k\left(\sigma_{1}+0\right)=p$ then we say that there is a degeneracy of (the nonzero) phases of order $p$ at $\sigma=\sigma_{1}$.

In this section we consider the case $p \leqq 2$. There are a priori three possibilities:

(6.1) $n_{-}\left(\sigma_{1}-0\right)-n_{-}\left(\sigma_{1}+0\right)=0$;

(6.2) $n_{-}\left(\sigma_{1}-0\right)-n_{-}\left(\sigma_{1}+0\right)=1$;

(6.3) $n_{-}\left(\sigma_{1}-0\right)-n_{-}\left(\sigma_{1}+0\right)=2$.

We first consider the case (6.2) and later on show that the cases (6.1), (6.3) do not occur.

We divide the case (6.2) into two subcases:

(6.4) $n_{-}\left(\sigma_{1}\right)=n_{-}\left(\sigma_{1}-0\right)-1$;

(6.5) $n_{-}\left(\sigma_{1}\right)=n_{-}\left(\sigma_{1}-0\right)$.

We first consider the subcase (6.4). Then one of the intervals $J_{i}\left(\sigma_{1}\right)$ does not occur. If $g_{2}(t)<0$ then this cannot be the last negative phase.

It is impossible that both $I_{i}\left(\sigma_{1}\right)$ and $I_{i+1}\left(\sigma_{1}\right)$ are not present, for then the degeneracy is of order $\geqq 3$. Thus we may assume that $I_{i}\left(\sigma_{1}\right) \neq 0$ (the case $I_{i+1}\left(\sigma_{1}\right) \neq 0$ can be treated in a similar manner). For simplicity of notation we shall take $i=1$.

We have to distinguish two cases:

(6.6) $I_{2}\left(\sigma_{1}\right) \neq \varnothing$;

(6.7) $I_{2}\left(\sigma_{1}\right)=\varnothing$.

We first consider the case (6.6) and denote by $I\left(\sigma_{1}\right)$ the interval on $t=\sigma_{1}$ connecting the end-point of $I_{1}\left(\sigma_{1}\right)$ to the initial-point of $I_{2}\left(\sigma_{1}\right)$. It is clear that $u=0$ on $I\left(\sigma_{1}\right)$.

We again consider two cases:

(6.8) $I\left(\sigma_{1}\right)$ is an interval $\left\{\left(x, \sigma_{1}\right) ; \delta_{1} \leqq x \leqq \delta_{2}\right\}$ with $\delta_{1}<\delta_{2}$;

(6.9) $I\left(\sigma_{1}\right)$ consists of one point, say $(\bar{x}, \sigma)$. 
LEMMA 8. Let (6.4), (6.6) hold. If $(6.8)$ holds, then $a\left(u\left(x, \sigma_{1}\right)\right)=0$ for almost all $x$, $\delta_{1} \leqq x \leqq \delta_{2}$.

The physical interpretation of the assertion of the lemma is that the interval $I\left(\sigma_{1}\right)$ is occupied with water at zero temperature.

Proof. For any point $\left(\bar{y}, \sigma_{1}\right)$ lying in the interior of $I\left(\sigma_{1}\right)$, there cannot exist a disc $V$ with center $\left(\bar{y}, \sigma_{1}\right)$ such that $u<0$ in $V \cap\left\{t<\sigma_{1}\right\}$. In fact, the existence of such a disc would contradict Lemma $1^{\prime}$.

It follows that for any $\left(\bar{y}, \sigma_{1}\right)$ in the interior of $I\left(\sigma_{1}\right)$ there exists a sequence $\left\{\left(y_{m}, t_{m}\right)\right\}$ with $y_{m} \rightarrow \bar{y}, t_{m} \nearrow \sigma_{1}$ such that $u\left(y_{m}, t_{m}\right) \geqq 0$ for all $m$. Since the points $(x, t)$ with $t<\sigma_{1}$ on which $u(x, t)=0$ are of the form $x=s_{i}(t)(1 \leqq i \leqq k-1)$ and since the sign of $u(x, t)$ alternates from one side of free boundary to the other, we can take the points $\left(y_{m}, t_{m}\right)$ such that $u\left(y_{m}, t_{m}\right)>0$. But then $u\left(x, t_{m}\right)=0$ on at least one of the intervals $b_{1} \leqq x \leqq y_{m}, y_{m} \leqq x \leqq s_{3}(t)$. Applying Lemma 4 we conclude that either $a\left(u\left(x, \sigma_{1}\right)\right)=0$ for almost all $x, \delta_{1} \leqq x<\bar{y}$, or $a\left(u\left(x, \sigma_{1}\right)\right)=0$ for almost all $x$, $\bar{y} \leqq x \leqq \delta_{2}$. Since this is true for any $\bar{y} \in\left(\delta_{1}, \delta_{2}\right)$, the assertion of the lemma follows.

We shall now prove

LEMMA 9. Let (6.4), (6.6), (6.8) hold. Then there exists an $\varepsilon>0$ such that $n_{+}(\sigma)=n_{+}\left(\sigma_{1}-0\right)-1, n_{-}(\sigma)=n_{-}\left(\sigma_{1}-0\right)-1$ if $\sigma_{1}<\sigma<\sigma_{1}+\varepsilon$. Furthermore, there are no zero intervals of the form (3.1) if $\sigma_{1}<\sigma<\sigma_{1}+\varepsilon$.

Proof. $u$ is a weak solution for $\sigma_{1}<t<T$ and $a\left(u\left(x, \sigma_{1}\right)\right)=0$ on $I\left(\sigma_{1}\right)$ (by Lemma 8). We claim that there exists a rectangle $R$ defined by $\delta_{1}-\delta<x<\delta_{2}+\delta, \sigma_{1}<t<\sigma_{1}$ $+\varepsilon$ such that $u>0$ in $R$. This can be proved by comparison, employing the corollary to Theorem 4. Indeed, we take smooth initial data $k(x)$ on $t=\sigma_{1}$ such that $k(x) \leqq 0, a(k(x)) \leqq a\left(u\left(x, \sigma_{1}\right)\right)$ outside an interval $\delta_{1}-\delta^{\prime}<x<\delta_{2}+\delta^{\prime}$ and $k(x) \geqq 0$, $k(x) \not \equiv 0, a(k(x))=\alpha_{1} k(x)$ in this interval. The boundary data are taken to be smooth negative functions, smaller than the corresponding boundary data of $u$.

By [1], there exists a classical solution $\left(w, \hat{s}_{1}, \hat{s}_{2}\right)$ to the new problem for $\sigma_{1}<t<\sigma_{1}+\delta^{\prime \prime}$, where $\hat{s}_{1}, \hat{s}_{2}$ are the free boundaries, and $\hat{s}_{1}\left(\sigma_{1}\right)=\delta_{1}-\delta^{\prime}, \hat{s}_{2}\left(\sigma_{1}\right)$ $=\delta_{2}+\delta^{\prime}$. By the maximum principle, $w(x, t)>0$ if $\hat{s}_{1}(t)<x<\hat{s}_{2}(t)$. Since $w$ is also a weak solution, the corollary to Theorem 4 shows that $u \geqq w$. Hence $u>0$ in the rectangle $R$ introduced above.

From the last statement and the continuity of $u$ it is clear that $I_{1}(\sigma)=I_{2}(\sigma)$ if $\sigma_{1}<\sigma<\sigma_{1}+\varepsilon$. Hence $n_{+}(\sigma)=n_{+}\left(\sigma_{1}\right)-1=n_{+}\left(\sigma_{1}-0\right)$.

The assertion $n_{-}(\sigma)=n_{-}\left(\sigma_{1}-0\right)-1$ now follows from Theorem 6 and the continuity of $u$. Finally, if there is a zero interval on $t=\sigma, \sigma_{1}<\sigma<\sigma_{1}+\varepsilon$, it must lie to the right of $I_{2}(\sigma)$ and the proof of Lemma 7 then applies. Thus we obtain a contradiction.

If instead of (6.8) we assume that (6.9) holds, then $s_{1}(t) \rightarrow \bar{x}, s_{2}(t) \rightarrow \bar{x}$ as $t \nearrow \sigma_{1}$. Furthermore, by introducing a weak solution (as before) for comparison with $u$, we get

COROLlaRY. Let (6.4), (6.6), (6.9) hold. Then the assertions of Lemma 9 are valid. 
We next consider the case where (6.6) is replaced by (6.7). We introduce $I\left(\sigma_{1}\right)$ as before and treat the cases (6.8), (6.9) separately.

LEMMA 10. Let (6.4), (6.7) hold. If (6.9) holds, then there exists a point $\left(\bar{y}, \sigma_{1}\right)$ with $\delta_{1} \leqq \bar{y} \leqq \delta_{2}$ such that

$$
\begin{aligned}
a\left(u\left(x, \sigma_{1}\right)\right) & =0 & & \text { if } \delta_{1}<x<\bar{y}, \\
& =-\alpha & & \text { if } \bar{y}<x<\delta_{2}
\end{aligned}
$$

for almost all $x$.

The physical interpretation of the assertion of the lemma is that the interval $\left\{\left(x, \sigma_{1}\right) ; \delta_{1} \leqq x<y\right\}$ is occupied with water at zero temperature, whereas the interval $\left\{\left(x, \sigma_{1}\right) ; \bar{y}<x<\delta_{2}\right\}$ is occupied with ice at zero temperature.

Proof. Set

$$
z_{1}=\limsup _{t / \sigma_{1}} s_{1}(t), \quad z_{2}=\liminf _{t / \sigma_{1}} s_{3}(t) .
$$

If $z_{1}=z_{2}$ then, by Lemma 4 , it follows that the assertion is valid with $\bar{y}=z_{1}$. It therefore suffices to show that the inequality $z_{1}<z_{2}$ is impossible. (Note that $z_{2}<z_{1}$ is impossible since it is easily seen to imply, by Lemma 4 , that $a\left(u\left(x, \sigma_{1}\right)\right)$ equals both 0 and $-\alpha$ for almost all $x$ in $\left(z_{2}, z_{1}\right)$.)

Suppose then that $z_{1}<z_{2}$ and take any $\bar{y}$ with $z_{1}<\bar{y}<z_{2}$. By the argument given at the beginning of the proof of Lemma 8 it follows that for any point $\bar{y} \in\left(\delta_{1}, \delta_{2}\right)$ there exists a sequence $\left\{\left(y_{m 1}, t_{m 1}\right)\right\}$ with $y_{m 1} \rightarrow \bar{y}, t_{m 1} \nearrow \sigma_{1}$ such that $u\left(y_{m 1}, t_{m 1}\right)>0$ for all $m$. Similarly there exists a sequence $\left\{\left(y_{m 1}, t_{m 2}\right)\right\}$ with $y_{m 2} \rightarrow \bar{y}, t_{m 2} \nearrow \sigma_{1}$ such that $u\left(y_{m 2}, t_{m 2}\right)<0$ for all $m$.

If we apply Lemma 4 we find that

$$
\begin{aligned}
a\left(u\left(x, \sigma_{1}\right)\right) & =0 & & \text { if } z_{1}<x<\bar{y}, \\
& =-\alpha & & \text { if } \bar{y}<x<z_{2},
\end{aligned}
$$

for almost all $x$. Since $\bar{y}$ is an arbitrary point in $\left(z_{1}, z_{2}\right)$, this is impossible.

LEMMA 11. Let (6.4), (6.7), (6.8) hold. Then there exists an $\varepsilon>0$ such that $n_{+}(\sigma)$ $=n_{+}\left(\sigma_{1}-0\right)-1, n_{-}(\sigma)=n_{-}\left(\sigma_{1}-0\right)-1$ if $\sigma_{1}<\sigma<\sigma_{1}+\varepsilon$. Furthermore, there are no zero intervals of the form (3.1) if $\sigma_{1}<\sigma<\sigma_{1}+\varepsilon$.

Proof. Since $n_{+}\left(\sigma_{1}\right)=n_{+}\left(\sigma_{1}-0\right)-1, n_{-}\left(\sigma_{1}\right)=n_{-}\left(\sigma_{1}-0\right)-1$ and each two phases of the same sign are separated, on $t=\sigma_{1}$, by a phase of the opposite sign, Theorem 6 and the continuity of $u$ imply that $n_{+}(\sigma)=n_{+}\left(\sigma_{1}-0\right)-1, n_{-}(\sigma)=n_{-}\left(\sigma_{1}-0\right)-1$ in sorile interval $\sigma_{1}<\sigma<\sigma_{1}+\varepsilon$.

The proof of Lemma 7 applies if there are no zero intervals $I$ of the form (3.1), $\sigma_{1}<\sigma<\sigma_{1}+\varepsilon$, lying to the right of $J_{2}(\sigma)$. Hence, that if there exists a zero interval $I$ of the form (3.1), $\sigma_{1}<\sigma<\sigma_{1}+\varepsilon$, then it must lie between $I_{1}(\sigma)$ and $J_{2}(\sigma)$. (Note that $I_{2}(\sigma)$ does not exist if $\sigma>\sigma_{1}$; in the situation of Lemma $9, I_{2}(\sigma)=I_{1}(\sigma)$ if $\sigma_{1}<\sigma<\sigma_{1}+\varepsilon$.) Denote the initial and end-points of $I$ by $\left(x_{0}(\sigma), \sigma\right)$ and $\left(x_{1}(\sigma), \sigma\right)$ respectively. 
We claim that

$$
x_{0}(\sigma) \nearrow \text { if } \sigma \nearrow \quad x_{1}(\sigma) \searrow \text { if } \sigma \nearrow .
$$

To prove (6.11) we first show that the projection of $I$ on $t=\sigma_{1}$ must be contained in the interval $I\left(\sigma_{1}\right)$. Suppose this is false, then by taking a subinterval of $I$ we may assume that its projection on $t=\sigma_{1}$ does not intersect $I\left(\sigma_{1}\right)$.

We can now proceed to derive a contradiction by employing the arguments of §3. We note that the maximal intervals $J_{y \mu}$ do not reach the line $t=\sigma_{1}$ and thus are either of positive end or of negative end (or both). Lemma 5 can be extended with some modifications in the proof, similar to those introduced in the proof of Lemma 7. In the present case we introduce monotone curves $\gamma_{1}, \tilde{\gamma}_{2}$ whose intersection with $t=\sigma, \sigma_{1} \leqq \sigma<\sigma_{1}+\varepsilon$, lies in $I_{1}(\sigma)$ and $J_{2}(\sigma)$ respectively. Further details may be omitted.

Having derived a contradiction to the assumption that the projection of $I$ does not lie in $I\left(\sigma_{1}\right)$, we next observe that the same proof shows also that the projection of the zero interval $I$ of the form (3.1) on the line $t=\sigma^{\prime}$, for any $\sigma_{1}<\sigma^{\prime}<\sigma$, must lie in the zero interval $I\left(\sigma^{\prime}\right)$ of $u$ which is located on $t=\sigma^{\prime}$ between $I_{1}\left(\sigma^{\prime}\right)$ and $J_{2}\left(\sigma^{\prime}\right)$. Since $\sigma, \sigma^{\prime}$ are arbitrary, (6.11) follows.

There are now three cases to consider:

(6.12) $\bar{y}=\delta_{1}$;

(6.13) $\bar{y}=\delta_{2}$;

(6.14) $\delta_{1}<\bar{y}<\delta_{2}$.

Suppose (6.12) holds. We shall prove that for any $\delta>0$ sufficiently small there exists a rectangle $R$ of the form $\delta_{1}+\delta<x<\delta_{2}, \sigma_{1}<t<\sigma_{1}+\delta^{\prime}$ with $\delta^{\prime}>0$ such that $u<0$ in $R$. This and (6.11) would then show that $x_{0}\left(\sigma_{1}\right)=x_{1}\left(\sigma_{1}\right)$, i.e. there are no zero intervals for $\sigma_{1}<\sigma<\sigma_{1}+\varepsilon$.

To construct $R$ as stated above, we employ the technique of comparison, via the corollary to Theorem 4. We construct a solution $w$ with initial data on $t=\sigma_{1}$ such that $a(w) \geqq a(u)$ on $t=\sigma_{1}$ and with boundary data larger than those of $u$. We can choose all these data to be sufficiently smooth and such that $w\left(x, \sigma_{1}\right) \leqq 0$, $w\left(x, \sigma_{1}\right) \not \equiv 0$ for $\bar{y}+\delta^{\prime \prime} \leqq x \leqq \delta_{2}+\delta^{\prime \prime}$ for some $\delta^{\prime \prime}>0$, and $w\left(x, \sigma_{1}\right)>0$ if $x<\bar{y}+\delta^{\prime \prime}$ or $x>\delta_{2}+\delta^{\prime \prime}$. The function $a(w)$ on $t=\sigma_{1}$ is chosen so that $a\left(w\left(x, \sigma_{1}\right)\right)=-\alpha$ if $w\left(x, \sigma_{1}\right)=0, \bar{y}+\delta^{\prime \prime} \leqq x \leqq \delta_{2}+\delta^{\prime \prime}$. There exists a classical solution $\left(w, \hat{s}_{1}, \hat{s}_{2}\right)$ for $\sigma_{1}<t<\sigma_{1}+\delta^{*}$, and its two free boundaries $x=\hat{s}_{1}(t), x=\hat{s}_{2}(t)$ satisfy: $\hat{s}_{1}\left(\sigma_{1}\right)=\bar{y}+\delta^{\prime \prime}$, $s_{2}\left(\sigma_{1}\right)=\delta_{2}+\delta^{\prime \prime}$. Furthermore, by the maximum principle, $w(x, t)<0$ if $\hat{s}_{1}(t)<x$ $<\hat{s}_{2}(t), \sigma_{1}<t<\sigma_{1}+\delta^{*}$. Since by the corollary to Theorem $4, u \leqq w$, we conclude that there exists a rectangle $R$ as above with $u<0$ in $R$.

The previous considerations obviously extend to the case where (6.12) is replaced by (6.13). If (6.14) holds, then we first have to construct a rectangle $R$ as in the case of (6.12) (and the construction is, in fact, the same as for that case) and then to construct a rectangle as in the case of (6.14).

The proof of Lemma 11 is thereby completed. 
If instead of (6.8) we assume that (6.9) holds, then $s_{1}(t) \rightarrow \bar{x}, s_{2}(t) \rightarrow \bar{x}, s_{3}(t) \rightarrow \bar{x}$, and Theorem 7 can be applied with $t=0$ replaced by $t=\sigma_{1}$. Hence,

LEMMA 11'. Let (6.4), (6.7), (6.9) hold. Then the assertions of Lemma 11 are valid.

We now consider the case where instead of (6.4) we assume that (6.5) holds. Thus, the number of negative phases on $t=\sigma_{1}$ is the same as on $t=\sigma$ for $\sigma<\sigma_{1}$. We claim that there must exist two negative phases $J_{i}, J_{i+1}$ on $\sigma=\sigma_{1}$ such that $u=0$ on the closed interval $I$ connecting the end-point of $J_{i}$ to the initial-point of $J_{i+1}$. Indeed, if this not true then from Theorem 6 and the continuity of $u$ we find that $n_{-}\left(\sigma_{1}+\varepsilon\right)=n_{-}\left(\sigma_{1}\right)$ if $\varepsilon$ is sufficiently small, which contradicts (6.2).

There are two cases to consider, namely, the case where $I$ is an interval of positive length and the case where $I$ consists of one point. It is clear that in the first case we have a situation similar to (6.4), (6.6), (6.8) with the roles of the negative and positive phases interchanged. If $I$ consists of one point then we have a situation similar to (6.4), (6.6), (6.9) with the roles of the negative and positive phases interchanged.

Having analyzed completely the case (6.2), we now turn to the case (6.1). If

$$
n_{+}\left(\sigma_{1}-0\right)-n_{+}\left(\sigma_{1}+0\right)=1 \text {, }
$$

then, by the previous results with the roles of the positive and negative phases interchanged, we conclude that (6.2) holds; a contradiction. Thus (6.1) cannot occur.

If (6.1) holds and

$$
n_{+}\left(\sigma_{1}-0\right)-n_{+}\left(\sigma_{1}+0\right)=2,
$$

then we again get a contradiction. In fact, we then must have $n_{+}\left(\sigma_{1}\right)=n_{+}\left(\sigma_{1}-0\right)-2$ (for otherwise the continuity of $u$ implies that (6.16) cannot hold). If the two positive phases which do not occur on $t=\sigma_{1}$ are not adjacent, then, the previous methods (of the case of (6.2)) show that (6.3) holds. If, on the other hand, they are adjacent then the negative phase lying between them cannot occur. Thus, in both cases (6.1) is contradicted.

We have thus proved that (6.1) cannot hold.

Finally, (6.3) also cannot hold. In fact, by arguing as in the case where (6.16) holds we find that if (6.3) holds then $n_{+}\left(\sigma_{1}-0\right)-n_{+}\left(\sigma_{1}+0\right) \geqq 1$. Thus the degeneracy is of order $p \geqq 3$, a contradiction.

We sum up the results of this section.

THEOREM 8. If the degeneracy $p=k\left(\sigma_{1}-0\right)-k\left(\sigma_{1}+0\right)$ is positive and $\leqq 2$, then $p=2$. There exists an $\varepsilon>0$ such that $n_{-}(\sigma)=n\left(\sigma_{1}-0\right)-1, n_{+}(\sigma)=n_{+}\left(\sigma_{1}-0\right)-1$ and such that there are no zero intervals of the form (3.1) whenever $\sigma_{1}<\sigma<\sigma_{1}+\varepsilon$. On $\sigma=\sigma_{1}$ there may be at most one zero interval and (if it exists) it is the union of two disjoint intervals (one of which may consist of just one point) such that $a\left(u\left(x, \sigma_{1}\right)\right)=0$ 
for almost all $x$ in one interval and $a\left(u\left(x, \sigma_{1}\right)\right)=-\alpha$ for almost all $x$ in the other interval.

We can now apply Theorem 7 with $t=0$ replaced by $t=\sigma$, for some $\sigma \in\left(\sigma_{1}, \sigma_{1}+\varepsilon\right)$. Denoting by $\sigma_{2}$ the largest number such that $k(\sigma)=k\left(\sigma_{1}+0\right)$ for all $\sigma_{1}<\sigma<\sigma_{2}$, we conclude that

COROLlaRY. There exist $k-3$ continuous functions $\tilde{s}_{i}(t)$, for $\sigma_{1} \leqq t<\sigma_{2}$, such that $b_{1}<\tilde{s}_{1}(t)<\cdots<\tilde{s}_{k-3}(t)<b_{2}$ and such that $u(x, t)>0$ if $\tilde{s}_{2 i}(t)<x<\tilde{s}_{2 i+1}, \sigma_{1}<t<\sigma_{2}$ and $u(x, t)<0$ if $\tilde{s}_{2 i-1}(t)<x<\tilde{s}_{2 i}(t)$ (here $\left.\tilde{s}_{0}(t) \equiv b_{1}, \tilde{s}_{k-2}(t) \equiv b_{2}\right)$.

The analog of (5.3) also holds.

The $\tilde{s}_{i}(t)$ are continuations of the $s_{j}(t)$ with appropriate indexing.

If the degeneracy at $\sigma=\sigma_{2}$ is again of order $\leqq 2$, then Theorem 8 and its corollary can obviously be extended with the role of $\sigma_{1}$ given to $\sigma_{2}$. This procedure can be further applied with $\sigma_{3}, \sigma_{4}, \ldots$, until we have gone through all the finite number, say $q$, of points of degeneracy (assuming that their degeneracy is always of order $\leqq 2)$. For $\sigma_{q} \leqq t<T$, the free boundaries, say $\hat{s}_{1}(t), \ldots, \hat{s}_{k-2 q}(t)$, are continuous functions and the analog of Theorem 7 holds in the interval $\left[\sigma_{q}, T\right)$.

7. Degeneracy of phases of any order. We shall study the case where $p=k\left(\sigma_{1}-0\right)$ $-k\left(\sigma_{1}+0\right) \geqq 3$, and set

$$
p_{0}=n_{-}\left(\sigma_{1}-0\right)-n_{-}\left(\sigma_{1}+0\right) .
$$

Then $p_{0} \geqq 2$ (since $p_{0} \leqq 1$ implies, by $\S 6$, that $p \leqq 2$ ).

There are two cases to be considered:

(7.2) $p_{0}=n_{-}\left(\sigma_{1}-0\right)-n_{-}\left(\sigma_{1}\right)$;

(7.3) $p_{0}>n_{-}\left(\sigma_{1}-0\right)-n_{-}\left(\sigma_{1}\right)$.

We shall consider here only the case (7.2), leaving out the necessary modifications in the case (7.3). Thus, there are $p_{0}$ negative phases $J_{i}(\sigma)\left(\sigma<\sigma_{1}\right)$ which disappear at $\sigma=\sigma_{1}$. We shall assume that their indices are consecutive since, otherwise, we may consider separately what happens at each block of consecutively degenerating phases. For the same reason we assume that each positive phase lying between two degenerating negative phases also disappears at $\sigma=\sigma_{1}$.

Thus, for some $i, J_{i}(\sigma), J_{i+1}(\sigma), \ldots, J_{i+p_{0}-1}(\sigma)$ and $I_{i+1}(\sigma), \ldots, I_{i+p_{0}-1}(\sigma)$ do not occur at $\sigma=\sigma_{1}$. We shall consider here only the case where $I_{i}\left(\sigma_{1}\right) \neq \varnothing$. The considerations in the case where $I_{i}\left(\sigma_{1}\right)=\varnothing$ are similar. For simplicity of notation we take $i=1$. Thus the phases $J_{1}\left(\sigma_{1}\right), \ldots, J_{p_{0}}\left(\sigma_{1}\right)$ and $I_{2}(\sigma), I_{3}(\sigma), \ldots, I_{p_{0}}(\sigma)$ disappear at $\sigma=\sigma_{1}$.

Note that a phase $I_{q}\left(\sigma_{1}\right)$ with $q>p_{0}+1$ cannot disappear at $\sigma=\sigma_{1}$ for, otherwise, by the considerations of $\S 6$ or those which follow below (with the roles of the positive and negative phases interchanged) it would follow that either $J_{q-1}(\sigma)=J_{q}(\sigma)$ or at least one of the phases $J_{q-1}(\sigma), J_{q}(\sigma)$ do not occur for $\sigma>\sigma_{1}, \sigma$ near $\sigma_{1}$, so that (7.1) is contradicted. 
We distinguish two cases:

(7.4) $I_{p_{0}+1}\left(\sigma_{1}\right) \neq \varnothing$;

(7.5) $I_{p_{0}+1}\left(\sigma_{1}\right)=\varnothing$.

We first consider the case (7.4) and denote by $I\left(\sigma_{1}\right)$ the interval connecting the end-point of $I_{1}\left(\sigma_{1}\right)$ to the initial-point of $I_{p_{0}+1}\left(\sigma_{1}\right)$.

We again distinguish two cases:

(7.6) $I\left(\sigma_{1}\right)$ consists of an interval $\left\{\left(x, \sigma_{1}\right) ; \delta_{1} \leqq x \leqq \delta_{2}\right\}$ with $\delta_{1}<\delta_{2}$;

(7.7) $I\left(\sigma_{1}\right)$ consists of one point, say, $\left(\bar{x}, \sigma_{1}\right)$.

Lemma 12. Assume that (7.4), (7.6) hold. Then there exist $2 q$ points $\left(z_{i}, \sigma_{1}\right)$ on $I\left(\sigma_{1}\right)$ with $\delta_{1} \leqq z_{1}<z_{2}<\cdots<z_{2 q} \leqq \delta_{2}, 1 \leqq q \leqq p_{0}-1$, such that

$$
\begin{array}{r}
a\left(u\left(x, \sigma_{1}\right)\right)=0 \quad \text { if } \delta_{1}<x<z_{1}, \text { if } z_{2 q}<x<\delta_{2}, \text { or if } z_{2 i}<x<z_{2 i+1} \\
\text { for some } i, 1 \leqq i \leqq q-1, \\
=-\alpha \quad \text { if } z_{2 i-1}<x<z_{2 i} \quad \text { for some } i, 1 \leqq i \leqq q
\end{array}
$$

for almost all $x$.

Proof. The free boundaries whose limit sets as $t \nearrow \sigma_{1}$ lie on $I\left(\sigma_{1}\right)$ are precisely $s_{1}(t), s_{2}(t), \ldots, s_{2 p_{0}}(t)$. Set

$$
z_{1}=\limsup _{t / \sigma_{1}} s_{1}(t), \quad z_{2 q}=z_{2 p_{0}}^{\prime}=\lim _{t / \pi \sigma_{1}} \inf s_{2 p_{0}}(t) .
$$

Then, by Lemma $4, a\left(u\left(x, \sigma_{1}\right)\right)=0$ for almost all $x$ in $\left(\delta_{1}, z_{1}\right)$ and in $\left(z_{2 q}, \sigma_{2}\right)$. Set

$$
z_{j}^{\prime}=\lim _{t / \sigma_{1}} \sup _{j}(t) \quad\left(2 \leqq j \leqq 2 p_{0}-1\right),
$$

and suppose that $z_{2}^{\prime}>z_{1}$. Note that for every sequence $\left\{\left(y_{m}, t_{m}\right)\right\}$ converging to some point $\left(\bar{y}, \sigma_{1}\right)$ with $z_{1}<\bar{y}<z_{2}^{\prime}, t_{m}<\sigma_{1}$, if $\left(y_{m}, t_{m}\right) \in J_{2}\left(t_{m}\right)$ then also, for any $\varepsilon>0, \varepsilon<\bar{y}-z_{1}$, the interval $\left\{\left(y, t_{m}\right) ; z_{1}+\varepsilon \leqq y \leqq y_{m}\right\}$ belongs to $J_{2}\left(t_{m}\right)$ if $m$ is sufficiently large. Using Lemma 4 we conclude that $a\left(u\left(x, \sigma_{1}\right)\right)=-\alpha$ for almost all $x$ with $z_{1}<x<\bar{y}$. Since $\bar{y}$ is an arbitrary point in $\left(z_{1}, z_{2}^{\prime}\right)$, the last assertion holds also if $\bar{y}=z_{2}^{\prime}$. We then take $z_{2}=z_{2}^{\prime}$ and proceed to the next interval. If $z_{2}^{\prime}=z_{1}$ then we proceed to the next interval directly.

If $z_{3}^{\prime}>z_{2}^{\prime}$ then we find, by the above reasoning, that $a\left(u\left(x, \sigma_{1}\right)\right)=0$ for almost all $x$ in the interval $z_{2}^{\prime}<x<z_{3}^{\prime}$. We then set $z_{3}=z_{3}^{\prime}\left(\right.$ or $z_{2}=z_{3}^{\prime}$ if $z_{2}^{\prime}=z_{1}$ ) and proceed to the next interval. If $z_{3}^{\prime}=z_{2}^{\prime}$ then we proceed to the next interval directly.

It is clear that in this way we arrive at (7.8) with some $q \leqq p_{0}$. Thus it remains to show that $q \neq p_{0}$. If $q=p_{0}$ then

$$
\delta_{1} \leqq z_{1}<z_{2}^{\prime}<z_{3}^{\prime}<\cdots<z_{2 p_{0}-1}^{\prime}<z_{2 p_{0}}^{\prime} \leqq \delta_{2} .
$$

Let $z_{1}<\bar{y}<z_{2}^{\prime}$. In view of Lemma $1^{\prime}$ there must exist a sequence $\left\{\left(y_{m}, t_{m}\right)\right\}$ with $y_{m} \rightarrow \bar{y}, t_{m} \nearrow \sigma_{1}$, such that $u\left(y_{m}, t_{m}\right)>0$ for all $m$. Hence $\left(y_{m}, t_{m}\right) \in I_{i}\left(t_{m}\right)$ for some $i \geqq 2$. The length of $I_{i}\left(t_{m}\right)$ must converge to 0 as $m \rightarrow \infty$ for, otherwise, using Lemma 4 we conclude that $a\left(u\left(x, \sigma_{1}\right)\right)=0$ for almost all $x$ in some interval containing $\bar{y}$, which is impossible. 
Next, $J_{i}\left(t_{m}\right)$ contains points $\left(\bar{y}_{m}, t_{m}\right)$ with $\bar{y}_{m} \rightarrow \bar{y}$. Hence the end-points $\left(\bar{y}_{m, i}, t_{m}\right)$ of $J_{i}\left(t_{m}\right)$ satisfy: lim sup $y_{m, i} \leqq z_{2}^{\prime}$ (since otherwise we find, by Lemma 4, that $a\left(u\left(x, \sigma_{1}\right)\right)=-\alpha$ for almost all $x$ in some subinterval of $\left.\left(z_{2}^{\prime}, z_{3}^{\prime}\right)\right)$.

Next we find that the end-points $\left(y_{m, i+1}, t_{m}\right)$ of $I_{i+1}\left(t_{m}\right)$ satisfy lim sup $y_{m, i+1} \leqq z_{3}^{\prime}$; the end-points $\left(\bar{y}_{m, i+1}, t_{m}\right)$ of $J_{i+1}\left(t_{m}\right)$ satisfy lim sup $\bar{y}_{m, i+1} \leqq z_{4}^{\prime}$, etc. Finally, the end-points $\left(\bar{y}_{m, p_{0}}, t_{m}\right)$ of $J_{p_{0}}\left(t_{m}\right)$ satisfy $\lim \sup \bar{y}_{m, p_{0}} \leqq z_{2 p_{0}-2 i+2}^{\prime}$. Since, however, $\lim \sup \bar{y}_{m, p_{0}} \geqq \lim \inf _{t / \sigma_{1}} s_{2 p_{0}}(t)=z_{2 p_{0}}^{\prime}$ and $i \geqq 2$, we get a contradiction.

In contrast with the case $p \leqq 2$, we now may possibly have zero intervals for $\sigma>\sigma_{1}$. The considerations of $\S 6$ show that if there is a zero interval $I(\sigma)$ of the form (3.1) with $\sigma_{1}<\sigma<\sigma_{1}+\varepsilon$ ( $\varepsilon$ sufficiently small so that no new degeneracy occurs) then there is in fact a zero region $\Delta_{\sigma}$ bounded by the curves

$$
t=\sigma_{1}, \quad t=\sigma, \quad x=x_{0}(t), \quad x=x_{1}(t) \quad\left(x_{0}(t)<x_{1}(t)\right)
$$

where $x_{0}(t)$ is monotone increasing and $x_{1}(t)$ is monotone decreasing. Furthermore,

$$
z_{1} \leqq x_{0}\left(\sigma_{1}\right), \quad x_{1}\left(\sigma_{1}\right) \leqq z_{2 q} .
$$

From Theorem 6 and the continuity of $u$ we also infer that there cannot exist two distinct zero intervals of the form (3.1) if $\sigma_{1}<\sigma<\sigma_{1}+\varepsilon$ where $\varepsilon$ is sufficiently small. Hence we actually have

$$
x_{0}\left(\sigma_{1}\right)=z_{1}, \quad x_{1}\left(\sigma_{1}\right)=z_{2 q} .
$$

To analyze the behavior of $u$ for $\sigma>\sigma_{1}$, let us assume at first, that there are no further degeneracies at any future time.

LEMMA 13. If a set $S$ defined by $z_{2 i-1}<x<z_{2 i}, \sigma_{1}<t<\sigma$ is contained in the zero region $\Delta_{\sigma}$, then $u(x, t)=0, a(u(x, t))=-\alpha$ in $S$ (for each $t$, almost everywhere in $\left.x\right)$. Similarly, if a set $S^{\prime}$ defined by $z_{2 i}<x<z_{2 i+1}, \sigma_{1}<t<\sigma$ is contained in $\Delta_{\sigma}$, then $u(x, t)=0, a(u(x, t))=0$ in $S^{\prime}$ (for each $t$, almost everywhere in $\left.x\right)$.

Proof. We only need to prove the assertions concerning $a(u)$. Now, from (1.9) it follows that the weak $t$-derivative of $a(u(x, t))$ in $S$ or in $S^{\prime}$ is zero. Since also $t \rightarrow a(u(x, t))$ is a weakly continuous function into $L^{2}(G)$, it follows that $a(u(x, t))$ is independent of $t$. Now use (7.8).

LEMMA 14. The function $x=x_{0}(\sigma)$ is strictly monotone decreasing. If it meets the line $x=z_{2}$ at $\sigma=\sigma_{1}^{*}$ then it is continuous in the interval $\sigma_{1}<\sigma<\sigma_{1}^{*}$, and, if $q>1$, it has a discontinuity of the first kind at $\sigma=\sigma_{1}^{*}$, with jump $z_{3}-z_{2}$ (provided $x_{0}(\sigma)$ is defined in some interval containing $\sigma_{1}^{*}$ ).

If the curve further meets the line $x=z_{4}$ at $\sigma=\sigma^{*}$, then it is continuous for $\sigma_{1}^{*}<\sigma<\sigma_{2}^{*}$ and has a jump $z_{5}-z_{4}$ at $\sigma=\sigma_{2}^{*}$, provided $q>2$. Similar results hold for $x_{1}(\sigma)$.

Proof. If $x_{0}(\sigma)$ is not strictly monotone, then $x_{0}\left(\sigma^{\prime}\right)=x_{0}\left(\sigma^{\prime \prime}\right)$ for some $\sigma_{1}<\sigma^{\prime}<\sigma^{\prime \prime}$. 
But then $x_{0}(\sigma)=x_{0}\left(\sigma^{\prime}\right)$ for $\sigma^{\prime} \leqq \sigma \leqq \sigma^{\prime \prime}$. Denote by $\Gamma$ the interval $\left\{(x, \sigma) ; x=x_{0}\left(\sigma^{\prime}\right)\right.$, $\left.\sigma^{\prime}<\sigma<\sigma^{\prime \prime}\right\}$, and by $N$ the intersection of a neighborhood of $\Gamma$ with $\left\{(x, t) ; x<x_{0}\left(\sigma^{\prime}\right)\right.$, $\left.\sigma^{\prime}<t<\sigma^{\prime \prime}\right\}$. Then $\alpha_{1} u_{t}=u_{x x}$ in $N$ and $u=0$ on $\Gamma$. By known theorems [2] $u_{x}$ is continuous up to the boundary $\Gamma$. From the weak form of (1.5) and from the fact that $u=0$ to the right of $\Gamma$ we also conclude that $u_{x}=0$ on $\Gamma$. Since, however, $u$ attains its minimum on $N \cup \Gamma$ on $\Gamma$, we have [2] $u_{x}<0$ on $\Gamma$, a contradiction.

We next prove that $x_{0}(\sigma)$ is continuous if $\sigma_{1}<\sigma<\sigma_{1}^{*}$. Suppose the assertion is false. Then there occurs a jump at some point $\bar{\sigma}, \sigma_{1}<\bar{\sigma}<\sigma^{*}$. Let $(\bar{x}, \bar{\sigma})$ be a point with $x_{0}(\bar{\sigma}-0)<\bar{x}<x_{0}(\bar{\sigma}+0)$ and let $V$ be a disc about $(\bar{x}, \bar{\sigma})$ with radius $<\left[x_{0}(\bar{\sigma}+0)-x_{0}(\bar{\sigma}-0)\right] / 2$. Take in (1.9) a $C^{\infty}$ function $\phi$ with support in $V$. Setting $V_{\varepsilon}=V-\{(x, t) \in V ;|t-\bar{\sigma}|>\varepsilon\}$, we have

$$
\lim _{\varepsilon \rightarrow 0} \iint_{V_{\varepsilon}}\left[u \phi_{x x}+a(u) \phi_{t}\right] d x d t=0,
$$

provided $V$ does not intersect the boundary of $\Omega_{T}$. If we now integrate by parts and then use the definition of $a(u)$ for $u>0$, Lemma 13 , and the continuity of $u$, we obtain

which is impossible.

$$
\int_{x_{0}(\delta-0)}^{x_{0}(\delta+0)} \alpha \phi(x, \bar{\sigma}) d x=0,
$$

Suppose next that $x_{0}\left(\sigma_{1}^{*}-0\right)=z_{2}$. The fact that $x_{0}(\sigma)$ has a jump at $\sigma=\sigma^{*}$ then follows by comparison (via the corollary to Theorem 4), employing some of the arguments appearing in the proof of Lemma 11.

So far, we have considered the case (7.6). If (7.7) holds then the situation is much simpler. In particular, no zero regions will develop.

Consider finally the case where instead of (7.4) we assume that (7.5) holds. Instead of the assertion (7.8) of Lemma 12, we now have

$$
\begin{aligned}
& a(u(x, t))=0 \quad \text { if } \delta_{1}<x<z_{1} \text { or if } z_{21}<x<z_{21+1} \text { for some } i, 1 \leqq i \leqq q-1 \text {, } \\
& =-\alpha \text { if } z_{2 q-1}<x<\delta_{2} \text { or if } z_{21-1}<x<z_{2 i} \text { for some } i, 1 \leqq i \leqq q-1 \text {; }
\end{aligned}
$$

here $q \leqq p$. Lemmas 13,14 remain valid.

We sum up most of the results obtained in this section:

THEOREM 9. Assume that there is only one degeneracy, that it occurs on $\sigma=\sigma_{1}$ in one block of phases, and that its order is $\geqq 3$. Then

$$
n_{-}\left(\sigma_{1}-0\right)=n_{-}(\sigma)+p_{0}, \quad n_{+}\left(\sigma_{1}-0\right)=n_{+}(\sigma)+\bar{p}_{0}(\sigma) \text { for } \sigma_{1}<\sigma<T,
$$

where either $\bar{p}_{0}(\sigma) \equiv p_{0}$ or there exists a $\bar{\sigma}, \sigma_{1}<\bar{\sigma}<\tau$, such that $\bar{p}_{0}(\sigma)=p_{0}-1$ if $\sigma_{1}<\sigma<\bar{\sigma}$ and $\bar{p}_{0}(\sigma)=p_{0}$ if $\sigma>\bar{\sigma}$. On $\sigma=\sigma_{1}$, there is at most one zero interval I. It can be broken into at most $p_{0}+p_{0}\left(\sigma_{1}+0\right)$ intervals such that in adjacent intervals $a\left(u\left(x, \sigma_{1}\right)\right)$ alternates its values from 0 to $-\alpha$ (its signature on the first and last intervals is the same as its signature on the intervals preceding and following $I$ respectively). 
If there is a zero region above $I$, then its lateral boundary is given by $x=x_{0}(\sigma)$, $x=x_{1}(\sigma)$. These functions satisfy the properties stated in Lemma 14. Finally, in the zero region, for each $t, a(u(x, t))=a\left(u\left(x, \sigma_{1}\right)\right)$ almost everywhere in $x$.

If there is another degeneracy in a different block of phases, at any time $\sigma \geqq \sigma_{1}$, then there may develop another zero region $\Delta^{\prime}$. In view of the monotonicity property of the lateral boundary of the zero regions, the two zero regions cannot intersect.

It is possible however that at some time $\sigma=\sigma_{2}$ there will occur a degeneracy that will involve the zero region of Theorem 9 with some other phases. Then the analysis of the situation is very similar to that considered before, except that now one of the two intervals bounding the new zero interval (on $\sigma=\sigma_{2}$ ) is itself a zero interval.

We shall not bother to state here in detail the most general situation that may occur. We only mention, in conclusion, two facts:

(i) Denote by $\tau_{1}, \tau_{2}, \ldots, \tau_{m}$ the points of degeneracy of the total number of phases (i.e. positive, negative, zero with $a(u) \equiv 0$, and zero with $a(u) \equiv-\alpha)$; then $m \leqq k-2$. In each interval $\tau_{i}<t<\tau_{i+1}\left(\tau_{0}=0, \tau_{m+1}=T\right)$ there exist continuous functions $x=\lambda_{j}(t)(j=1, \ldots, j(i) ; j(i) \searrow$ if $i \nearrow)$ with $\lambda_{1}(t) \equiv b_{1}, \lambda_{j(i)}(t) \equiv b_{2}, \lambda_{1}(t)<$ $\lambda_{2}(t)<\cdots<\lambda_{j(i)}(t)$, such that the following holds:

In each region defined by $\lambda_{j}(t)<x<\lambda_{j+1}(t), \tau_{i}<t<\tau_{i+1}$ exactly one of the following situations is valid: $(\alpha) u(x, t)>0 ;(\beta) u(x, t)<0 ;(\gamma) u(x, t) \equiv 0$ and $a(u(x, t)) \equiv 0$, and $(\delta) u(x, t) \equiv 0$ and $a(u(x, t))=-\alpha$.

(ii) Each set $G=\left\{t=\tau_{i}\right\}$ consists of a finite number of intervals $\Pi_{j}(j=1, \ldots$, $j^{*}(i) ; j^{*}(i) \searrow$ if $\left.i \nearrow\right)$ such that on each $\Pi_{j}$ precisely one of the cases $(\alpha),(\beta),(\gamma)$, $(\delta)$ of (i) is valid.

\section{Miscellaneous remarks.}

8.1. We explain the gap which occurs in the proof of existence of classical solutions for the two-phase Stefan problem, as given by Rubinstein [4]. He proves that if $t_{0}<t_{1}<\cdots<t_{n}<\cdots, t_{n} \rightarrow t_{*}$ and if a classical solution exists for $t<t_{*}$ and $(-1)^{k} d s(t) / d t \geqq 0$ in $\left(t_{k}, t_{k+1}\right)$, and if, finally, $\left|\partial u_{i}(x, t) / \partial x\right| \leqq \beta(i=1,2)$ for $x \in G$, $t=t_{0}$ (where $\beta$ is sufficiently large, say, $\beta \geqq \beta_{0}$, and $\beta_{0}$ depends only on the data), then $\left|\partial u_{i}(x, t) / \partial x\right| \leqq \beta$ for $x \in G, t_{0} \leqq t<t_{*}$.

This result, however, does not yet prove that a solution, if existing for all $t<\sigma$, can be continued beyond $t=\sigma$.

To show this, we introduce a function $f(t)(0 \leqq t \leqq 1)$ defined as follows: at each point $\tau_{\alpha}$ of the Cantor set $S$ its value is zero, and in each open interval $\left(\tau_{\alpha}, \tau_{\beta}\right)$ lying outside $S$, whose end-points $\tau_{\alpha}, \tau_{\beta}$ belong to $S$, it is defined by

$$
\begin{aligned}
f(t) & =t-\tau_{\alpha} & & \text { if } \tau_{\alpha}<t<\tau_{\alpha}+\left(\tau_{\beta}-\tau_{\alpha}\right) / 3, \\
& =t-\tau_{\beta} & & \text { if } \tau_{\alpha}+2\left(\tau_{\beta}-\tau_{\alpha}\right) / 3<t<\tau_{\beta},
\end{aligned}
$$

and in the interval $\tau_{\alpha}+\left(\tau_{\beta}-\tau_{\alpha}\right) / 3 \leqq t \leqq \tau_{\alpha}+2\left(\tau_{\beta}-\tau_{\alpha}\right) / 3 f(t)$ is the linear function with values $\left(\tau_{\beta}-\tau_{\alpha}\right) / 3$ and $-\left(\tau_{\beta}-\tau_{\alpha}\right) / 3$ at the initial- and end-points respectively. 
Since $|f(t)| \leqq \operatorname{dist}(t, S)$, it is easily seen that $f(t)$ is a continuous function for $0 \leqq t \leqq 1$. Note that if $\tau_{\alpha} \in S$ then $f(t)$ has an infinite number of oscillations in every interval $\left(\tau_{\alpha}, \tau_{\alpha}+\varepsilon\right)$ and $\left(\tau_{\alpha}-\varepsilon, \tau_{\alpha}\right)$.

Going back to Rubinstein's work, all one knows about the smoothness of $d s / d t$ is that this function is continuous. If, then, this function coincides, for $\sigma-1<t<\sigma$, with the function $f(t+1-\sigma)$, then the procedure of Rubinstein fails to yield a continuation of the solution across $t=\sigma$. In fact whenever $t_{0}$ is a point of the form $\tau_{\alpha}+1-\sigma$ (and there are such points arbitrarily close to $\sigma$ ) then there does not exist a sequence $\left\{t_{n}\right\}$ as above. The situation cannot be remedied by replacing $t_{0}$ by $t_{0}+\varepsilon$ and then trying to take $\varepsilon \rightarrow 0$. In fact, if $t_{0}+\varepsilon$ is not in $S$, Rubinstein's method yields extension of the bound on $u_{x}(x, t)$ from $t=t_{0}+\varepsilon$ up to $t=t_{0}+\varepsilon+\delta(\varepsilon)$ and $\delta(\varepsilon) \rightarrow 0$ as $\varepsilon \rightarrow 0$.

It is thus clear that the gap in [4] is of a fundamental nature. It is therefore not known whether classical solutions do in fact exist. This restricts the meaningfulness of the asymptotic results in [4], since the solutions considered there are classical ones.

8.2. Generalizations. All the results of this paper extend to the case where the equations in (1.1) are replaced by the more general equations

$$
\frac{\partial u_{i}}{\partial t}=\beta_{i} a(x, t) \frac{\partial^{2} u_{i}}{\partial x^{2}}+\beta_{i} b(x, t) \frac{\partial u}{\partial x}+c^{i}(x, t) u \quad(i=1,2)
$$

where $c^{i}(x, t) \leqq 0$ and $a, b, c^{t}$ satisfy some smoothness properties. Indeed, as mentioned in [3], Theorems 1, 2 certainly extend to these equations. A review of the previous sections now shows that all the results extend to the case of (1.1) with trivial modifications.

Note that the methods of [4] do not apply to equations as general as (8.1).

The results of the $\$ \$ 2-4$ extend without (essentially) any change in the proofs to the case where $s(0)=b_{1}$ or $s(\theta)=b_{2}$. The same is true for the remaining sections (with $s_{1}(0)=b_{1}$ or $s_{k}(0)=b_{2}$ ).

If, on $t=0$, one of the functions $h_{t}(x)$ is identically zero, then all the results of the previous sections remain valid. In fact, the situation is similar (though much simpler) to the situation for $\sigma>\sigma_{1}, \sigma$ near $\sigma_{1}$ (where $t=\sigma_{1}$ is the first line of degeneracy).

If the boundary conditions (1.2) are replaced by

$$
\lambda_{i}\left(\partial u_{i}\left(b_{i}, t\right) / \partial x\right)+\mu_{i} u_{i}\left(b_{i}, t\right)=g_{i}(t) \quad\left(\lambda_{i} \geqq 0, \mu_{i} \geqq 0, \lambda_{i}^{2}+\mu_{i}^{2}>0\right),
$$

then under suitable conditions (on the $g_{i}$ or on the magnitude of $T$ ) Theorems 1,2 remain true. Also the results of this paper are then valid (with minor modifications in the proofs).

8.3. Remark. One can use Theorem 2 and the corollary to Theorem 4 in order to obtain information on the free boundaries. For instance, if $g_{1}(t) \geqq \gamma_{1}>0$, $g_{2}(t) \geqq \gamma_{2}>0$ for all $t>0$, then we can compare this problem with another problem 
for which $g_{1}(t) \equiv \gamma_{1}, g_{2}(t) \equiv \gamma_{2}$ for all $t>1$ (thereby using the corollary to Theorem 4). For the new problem we may apply Theorem 2 and conclude, in particular, that the solution is $\geqq \varepsilon_{0}>0$ if $t$ is sufficiently large, say, $t \geqq T_{0}$. Hence, all the free boundaries disappear before $t=T_{0}$.

If, on the other hand, $g_{2}(t) \leqq \gamma_{2}<0$ and the conditions of Theorem 2 on $g(t)$ hold, then the assertion (1.14) is true for whichever free boundaries $x=s_{i}(t)$ that exist for all $t$ sufficiently large.

Added in proof. (I) Recently there appeared a paper by J. Douglas, J. R. Cannon and C. D. Hill, A multi-boundary Stefan problem and the disappearance of phases, J. Math. Mech. 17 (1967), 21-34. In this paper the authors prove the existence of a classical solution for the 3-phase Stefan problem with initial values $0 \leqq h_{1}(x) \leqq K_{1},-K_{2} \leqq h_{2}(x) \leqq 0,0 \leqq h_{3}(x) \leqq K_{1}$ in intervals $\left(-\infty, d_{2}\right),\left(d_{2}, d_{3}\right)$ and $\left(d_{3}, \infty\right)$ respectively, satisfying

$$
K_{1}+K_{2}<\alpha \quad(\alpha \text { as in (1.5)). }
$$

Their method extends to any number of phases as well as to the case where the infinite interval $(-\infty, \infty)$ is replaced by a finite interval $\left(b_{1}, b_{2}\right)$. The crucial step in their proof is the derivation of an a priori bound on the functions $\partial u_{t} / \partial x$ at the free boundaries (or, equivalently, on the functions $d s_{j} / d t$ ). This bound is derived by use of the maximum principle. It is in the derivation of this bound that the assumption (*) enters. It may be noted that, once this a priori bound is derived, the existence and uniqueness of a classical solution can be established also by the method of Friedman [1].

(II) Since this manuscript went to the printer we have noticed several papers in the Chinese literature dealing with the 2-phase Stefan problem. These are:

( $\alpha$ ) Chiang Li-Shang, The two-phase Stefan problem. II, Chinese Math.-Acta 5 (1964), 36-53 [Acta Math. Sinica 14 (1964), 33-49],

$(\beta) \longrightarrow$ Existence and differentiability of the solution of a two-phase Stefan problem for quasilinear parabolic equations, Chinese Math.-Acta 7 (1965), 481-496 [Acta Math. Sinica 15 (1965), 749-764], and several other articles quoted in the above papers, These papers claim to have proven existence of a classical solution for any finite $t$-interval. However they are based on a false derivation of a priori bounds on $\partial u_{1} / \partial x, \partial u_{2} / \partial x$ and, thus, the "proofs" they provide are invalid. We shall briefly explain the nature of the crucial mistake, as it occurs, say, in the article $(\alpha)$.

The author indicates (in $(\alpha)$, p. 45) that if $\partial u_{1} / \partial x$, considered in $0 \leqq x \leqq s(t)$, $0 \leqq t \leqq T$, takes its minimum at a point $\left(s\left(t^{*}\right), t^{*}\right)$ on the free boundary, then $d s\left(t^{*}\right) / d t>0$. Similarly, if $\partial u_{2} / \partial x$ takes its minimum at a point $(s(\bar{t}), \bar{t})$ on the free boundary, then $d s(\bar{t}) / d t<0$. Next he concludes that each of the functions $\partial u_{i} / \partial x$ $(i=1,2)$ cannot take its minimum at some point of the free boundary, by tacitly assuming that $t^{*}=\bar{t}$. This assumption, however, is not only unfounded but should also be generally false. 


\section{REFERENCES}

1. A. Friedman, Free boundary problems for parabolic equations. I. Melting of solids, J. Math. and Mech. 9 (1959), 499-518.

2. - Partial differential equations of parabolic type, Prentice-Hall, Englewood Cliffs, N. J., 1964.

3. - The Stefan problem in several space variables, Trans. Amer. Math. Soc. 132 (1968), 51-87.

4. L. I. Rubinstein, Two-phase Stefan problem on a segment with one-phase initial state of thermoconductive medium, UCen. Zap. Lat. Gos. Univ. Stuðki 58 (1964), 111-148.

\section{NORTHWESTERN UNIVERSITY, \\ Evanston, IllinoIs}

TEL-AVIV UNIVERSITY,

TeL-Aviv, ISRAEL 